\title{
Hidden Symmetries of Lax Integrable Nonlinear Systems
}

\author{
Denis Blackmore ${ }^{1}$, Yarema Prykarpatsky ${ }^{2}$, Jolanta Golenia ${ }^{3}$, Anatoli Prykapatski ${ }^{3}$ \\ ${ }^{1}$ Department of Mathematical Sciences, New Jersey Institute of Technology, Newark, USA \\ ${ }^{2}$ Department of Mathematics, Agriculture University, Krakow, Poland \\ ${ }^{3}$ Department of Applied Mathematics, AGH University of Science and Technology, Krakow, Poland \\ Email: deblac@m.njit.edu,yarpry@gmail.com,golenia@agh.edu.pl,prykanat@ua.fm \\ Received April 29, 2013; revised May 29, 2013; accepted June 7, 2013
}

Copyright (C) 2013 Denis Blackmore et al. This is an open access article distributed under the Creative Commons Attribution License, which permits unrestricted use, distribution, and reproduction in any medium, provided the original work is properly cited.

\begin{abstract}
Recently devised new symplectic and differential-algebraic approaches to studying hidden symmetry properties of nonlinear dynamical systems on functional manifolds and their relationships to Lax integrability are reviewed. A new symplectic approach to constructing nonlinear Lax integrable dynamical systems by means of Lie-algebraic tools and based upon the Marsden-Weinstein reduction method on canonically symplectic manifolds with group symmetry, is described. Its natural relationship with the well-known Adler-Kostant-Souriau-Berezin-Kirillov method and the associated $R$-matrix method $[1,2]$ is analyzed in detail. A new modified differential-algebraic approach to analyzing the Lax integrability of generalized Riemann and Ostrovsky-Vakhnenko type hydrodynamic equations is suggested and the corresponding Lax representations are constructed in exact form. The related bi-Hamiltonian integrability and compatible Poissonian structures of these generalized Riemann type hierarchies are discussed by means of the symplectic, gradientholonomic and geometric methods.
\end{abstract}

Keywords: Lie-Algebraic Approach; Marsden-Weinstein Reduction Method; R-Matrix Structure; Poissonian Manifold; Differential-Algebraic Methods; Gradient Holonomic Algorithm; Lax Integrability; Symplectic Structures; Compatible Poissonian Structures; Lax Representation

\section{Introduction}

It is well known that hidden symmetry properties, related to symplectic, differential-geometric, differential-algebraic (D-A) or analytical structures of nonlinear Hamiltonian dynamical systems on functional manifolds, such as an infinite hierarchy of conservation laws and compatible Poissonian structures, often give rise to their Lax integrability. This fact was extensively worked out by many researchers during the past half century and a very powerful so called inverse Lie-algebraic orbit method [1,3-6] of constructing hierarchies of a priori Lax integrable nonlinear dynamical systems was devised. A related direct problem of retrieving these hidden intrinsic symmetries for a priori given well posed nonlinear dynamical system, which are suspected to be Lax integrable, proved to be a very complicated task, whose solution is still far from being solved. Among different approaches to coping with it one can mention, for instance, the classical Kowalewskaya-Painlevé method and its modifications, the Mikhaylov-Shabat [7] recursion operator method, based on analyzing the Lie-Backlund symmetries and some other techniques, which appeared to be reasonably effective in diverse applications, especially for classifying nonlinear integrable dynamical systems possessing special structure. Recently, when studying integrability properties of infinite so called Riemann type hydrodynamical hierarchies, a new direct approach to testing the Lax integrability of a priori given nonlinear dynamical systems with special structure, based on treating the related symplectic and differential-algebraic structures of differentiations, was suggested [8] and devised in [9]. By means of this technique the direct integrability problem was effectively reduced to the classical one of finding the corresponding compatible representations in suitably constructed differential rings.

Concerning the inverse Lie-algebraic orbit method, as its name suggests, it consists $[1,3,6,10-12]$ in studying invariant orbits of the coadjoint group $\hat{G}$ action on a specially chosen element $l \in \mathcal{G}^{*}$, where $\mathcal{G}^{*}$ is the conjugate space to the Lie algebra $\mathcal{G}$ of a suitably chosen, in general formal, group $\hat{G}$. In other words, the main Lie-algebraic essence of this approach consists in considering functional invariance and related symplectic properties of these extended orbits in $\mathcal{G}^{*}$, generated by 
the given element $l \in \mathcal{G}^{*}$ and inherited from the standard Lie algebra structure of $\mathcal{G}$.

From this point of view, subject to this extension scheme of constructing a priori Lax integrable dynamical systems, it was natural to search for another way of constructing such systems, but based on a suitably chosen reduction construction of the corresponding coadjoint group $\hat{G}$ action on the general element $l \in \mathcal{G}^{*}$. Happily, in modern symplectic geometry such a reduction method was well developed many years ago by Marsden and Weinstein $[13,14]$ and effectively applied to studying integrability properties of some nonlinear dynamical systems $[15,16]$ on finite-dimensional symplectic manifolds. Thus, a next step, consisting in developing this Marsden-Weinstein reduction method and applying it to the case of infinite-dimensional dynamical systems on functional manifolds, was quite natural and effectively realized in [17]. The latter, in particular, made it possible to substantially generalize results of [18] and apply them to studying a new physically feasible and important model in modern quantum physics. As all of the topics, mentioned above and recently studied in our publications, are closely connected to each other, we tried in this work to review those main essentially used analytical, Lie-algebraic and differential-algebraic structures which proved to be algorithmically effective for studying Lax integrability of nonlinear dynamical systems on functional manifolds.

As an important example of applying these recently devised techniques, a new generalized Riemann type hydrodynamic system is studied by means of a novel combination of symplectic and differential-algebraic tools. A compatible pair of polynomial Poissonian structures, a Lax representation and a related infinite hierarchy of conservation laws are constructed. Also analyzed is the complete Lax integrability of the important (for applications) Ostrovsky-Vakhnenko Equation, studied by means of symplectic, gradient-holonomic and differential-algebraic tools. A compatible pair of polynomial Poissonian structures, Lax representations and related infinite hierarchies of conservation laws are also presented.

\section{Lax Integrability via Marsden-Weinstein Reduction and the AKS-BK and R-Matrix Approaches}

\section{Loop Group, Canonically Symplectic Manifold and Hamiltonian Action}

As it is well-known $[1,6,13,14]$, the most popular canonically symplectic manifolds are supplied by cotangent spaces $M:=T^{*}(P)$ to some "coordinates" phase spaces $P$, which can often possess additional symmetry properties. If this symmetry can be identified with a Lie group
$\tilde{G}$ action on the phase space $P$ and its natural extension on the whole manifold $M$ proves to be symplectic and even more, Hamiltonian, the Marsden-Weinstein reduction method $[10,13]$ makes it possible to construct new Hamiltonian flows on the smaller invariant reduced phase space $\bar{M}_{\xi}:=M_{\xi} / G_{\xi}$ subject to the group invariant constraint $p:=\xi \in \mathcal{G}^{*}$ for some specially chosen element $\xi \in \mathcal{G}^{*}$, where $p: M \rightarrow \mathcal{G}^{*}$ is the related momentum mapping on the symplectic manifold $M$ and $\mathcal{G}^{*}$ is the adjoint space to the Lie algebra $\mathcal{G}$ of the group Lie $\tilde{G}$.

As the corresponding Hamiltonian flows on the reduced phase space $\bar{M}_{\xi}$ often possess very interesting properties important for applications in many branches of mathematics and physics, they were topics of many investigations during the past decades. As a result of our interest in the mathematical properties of the Lax integrable flows, we observed that their modern Lie algebraic description by means of the Hamiltonian group action classical Lie-Poisson-Adler-Kostant-Berezin-Kirillov scheme on the adjoint space $\hat{\mathcal{G}}^{*}$ to the Lie algebra $\hat{\mathcal{G}}$ of a suitably chosen group $G$ is a natural consequence of applying the Marsden-Weinstein reduction method to the canonical symplectic phase space $M=T^{*}(P)$. The basis space $P$, has to be a specially chosen Lie algebra $\mathcal{G}$ with the naturally related Hamiltonian group $\tilde{G}$ action on the symplectic phase space $M$. Moreover, such classical integrability theory ingredients as $R$-structures [19] and the related commutation properties of the related transfer matrices are also naturally retrieved from the Marsden-Weinstein reduction method via the scheme specified above.

Consider a complex matrix Lie group $G=S L(v ; \mathbb{C})$, $v \in \mathbb{Z}_{+}$, its Lie algebra $\mathcal{G}$, and a related $[1,4,6]$ formal loop group $\tilde{G} \subset C^{\infty}\left(\mathbb{S}^{1} ; \operatorname{Hol}(\mathbb{C} ; G)\right)$ of $G$-valued functions on the circle $\mathbb{S}^{1}$, meromorphically depending on the complex parameter $\lambda \in \mathbb{C}$. Its Lie algebra $\tilde{\mathcal{G}}$ can be viewed as the completion

$$
\tilde{\mathcal{G}}=\bigcup_{n \in \mathbb{Z}}\left\{\sum_{j=-\infty}^{n} \tilde{X}_{j} \lambda^{j}: \tilde{X}_{j} \in C^{\infty}\left(\mathbb{S}^{1} ; \mathcal{G}\right)\right\} .
$$

By the standard procedure $[1,10]$ one can construct the centrally extended current algebra $\hat{\mathcal{G}}:=\tilde{\mathcal{G}} \oplus \mathbb{C}$, on which the adjoint loop group $\tilde{G}$-action is defined: for any $g \in \tilde{G}$

$$
g:(T, c) \rightarrow\left(g T g^{-1}, c+\left(g^{-1} g_{x}, T\right)_{-1}\right) .
$$

Here $(T, c) \in \hat{\mathcal{G}}$ and $(\cdot, \cdot)_{-1}: \tilde{\mathcal{G}} \times \tilde{\mathcal{G}} \rightarrow \mathbb{C}$ is the following nondegenerate symmetric scalar product on $\tilde{\mathcal{G}}$ :

$$
(A, B)_{-1}:=\operatorname{res} \int_{0}^{2 \pi} \operatorname{tr}(A(x ; \lambda) B(x ; \lambda))=(B, A)_{-1},
$$

for any $A, B \in \tilde{\mathcal{G}}$. The scalar product (3) is ad-invariant, that is 


$$
(A,[B, C])_{-1}=([A, B], C)_{-1}
$$

for any elements $A, B$ and $C \in \tilde{\mathcal{G}}$.

Define now the canonically symplectic phase space $M:=T^{*}(\hat{\mathcal{G}}) \simeq\left(\hat{\mathcal{G}}, \hat{\mathcal{G}}^{*}\right)$ with the corresponding Liouville 1-form on $M$ :

$$
\alpha^{(1)}(T, c ; l, k)=(l, \mathrm{~d} T)_{-1}+k \mathrm{~d} c,
$$

whose exterior derivative gives the symplectic structure on the functional manifold $M$ :

$$
\begin{aligned}
\omega^{(2)}(T, c ; l, k) & :=\mathrm{d} \alpha^{(1)}(T, c ; l, k) \\
& =(\mathrm{d} l, \wedge \mathrm{d} T)_{-1}+\mathrm{d} k \wedge \mathrm{d} c .
\end{aligned}
$$

Similarly to (2) one can naturally extend the group $\tilde{G}$-action on the whole phase space $M$, having

$$
g:(l, k) \rightarrow\left(g l g^{-1}-k g_{x} g^{-1}, k\right)
$$

for any $(l, k) \in \hat{\mathcal{G}}^{*}$ and $g \in \tilde{G}$ as the corresponding co-adjoint action of the current group $\tilde{G}$ to the adjoint linear space $\hat{\mathcal{G}}^{*}$. The following lemma is almost selfevident.

Lemma 1 The $\tilde{G}$-group action (2) and (7) on the symplectic phase space $M$ is symplectic and Hamiltonian.

It is easy to check that the canonical Liouville 1-form (5) on the manifold $M$ is $\tilde{G}$-invariant:

$$
\begin{aligned}
& g^{*} \alpha^{(1)}(T, c ; l, k) \\
& =\left(g l g^{-1}-k g_{x} g^{-1}, g \mathrm{~d} T g^{-1}\right)_{-1} \\
& +k\left(\mathrm{~d} c+\left(g^{-1} g_{x}, \mathrm{~d} T\right)_{-1}\right) \\
& =\left(g l g^{-1}, g \mathrm{~d} T g^{-1}\right)-k\left(g_{x} g^{-1}, g \mathrm{~d} T g^{-1}\right)_{-1} \\
& +k \mathrm{~d} c+k\left(g^{-1} g_{x}, \mathrm{~d} T\right)_{-1} \\
& =\left(l, g^{-1} g \mathrm{~d} T g^{-1} g\right)_{-1}-k\left(g^{-1} g_{x} g^{-1} g, \mathrm{~d} T\right)_{-1} \\
& +k \mathrm{~d} c+k\left(g^{-1} g_{x}, \mathrm{~d} T\right)_{-1} \\
& =(l, \mathrm{~d} T)_{-1}+k \mathrm{~d} c=\alpha^{(1)}(T, c ; l, k) .
\end{aligned}
$$

From (8), owing to the expression (6), one obtains the symplectic form invariance

$$
g^{*} \omega^{(2)}(T, c ; l, k)=\omega^{(2)}(T, c ; l, k)
$$

for any element $(T, c ; l, k) \in M$.

To define the Hamiltonian $G$-action on the symplectic manifold $M$ we take the group flow $g(t):=\exp (t X)$ for $t \in \mathbb{R}, X \in \tilde{\mathcal{G}}$, and find the generated vector field $K_{X}: M \rightarrow T(M)$ on the phase space $M$ :

$$
\begin{aligned}
& K_{X}(T, c ; l, k) \\
& :=\frac{\mathrm{d}}{\mathrm{d} t}\left(g(t) T g(t)^{-1}, c+\left(g(t)^{-1} g_{X}(t), T\right)_{-1} ;\right. \\
& \left.\quad g(t) \lg (t)^{-1}-k g_{x}(t) g(t)^{-1}, k\right)\left.\right|_{t=0} \\
& =\left([X, T],\left(X_{x}, T\right)_{-1} ;[X, l]-k X_{x}, 0\right),
\end{aligned}
$$

by a Hamiltonian function $H_{X}: M \rightarrow \mathbb{C}$ owing to the canonical relationship $-\mathrm{d} H_{X}=i_{K_{X}} \omega^{(2)}$ :

$$
\begin{aligned}
-\mathrm{d} H_{X} & =-(\partial H / \partial l, \mathrm{~d} l)_{-1}-\left(\partial H_{X} / \partial T, \mathrm{~d} T\right)_{-1} \\
& +\partial H_{X} / \partial k \mathrm{~d} k+\partial H_{X} / \partial c \mathrm{~d} c \\
& =\left([X, l]-k X_{X}, \mathrm{~d} T\right)_{-1}-(\mathrm{d} l,[X, T])_{-1} \\
& -\left(X_{X}, T\right)_{-1} \mathrm{~d} k .
\end{aligned}
$$

As a consequence of (11), one obtains

$$
\begin{aligned}
& \partial H_{X} / \partial l=[X, T], \\
& \partial H_{X} / \partial T=k X_{X}-[X, l], \\
& \partial H_{X} / \partial k=\left(X_{X}, T\right)_{-1}, \\
& \partial H_{X} / \partial c=0
\end{aligned}
$$

for any point $(T, k ; l, c) \in M$. From (12) it follows that

$$
H_{X}=\left([T, l]-k T_{x}, X\right)_{-1}:=(p(T, c ; l, k), X)_{-1},
$$

is linear with respect to the generator element $X \in \tilde{\mathcal{G}}$. This means that the loop group $\tilde{\mathcal{G}}^{*}$ action on the symplectic manifold $M$ is Hamiltonian by definition $[12,13] . \triangleright$

The corresponding mapping $p: M \rightarrow \tilde{\mathcal{G}}^{*}$, where

$$
p(T, c ; l, k)=[T, l]-k T_{x},
$$

is called the momentum mapping $[10,12,13]$ which can be constrained to be fixed for further applications to the phase space $M$ in the Marsden-Weinstein reduction procedure [13].

Let us describe in detail the related symplectic structure on the $\xi$-level submanifold

$$
M_{\xi}:=\left\{(T, c ; l, k) \in M:[T, l]-k T_{x}=\xi \in \tilde{\mathcal{G}}^{*}\right\}
$$

for a fixed element $\xi \in \tilde{\mathcal{G}}^{*}$. As a more natural case we take that $\xi=0 \in \tilde{\mathcal{G}}^{*}$. The corresponding isotropy group $\tilde{G}_{\xi}=\tilde{G}$, as $\left.\operatorname{Ad}_{g}^{*} \xi\right|_{\xi=0}=0$ holds for any element $g \in \tilde{G}$.

To proceed further, we need some additional properties of the submanifold $M_{\xi} \subset M$, which we describe next.

\section{Marsden-Weinstein Reduction and Poisson Brackets}

In this section we shall be interested in describing the submanifold $M_{\xi} \subset M$ parameterized by the points of 
the reduced phase space $\bar{M}_{\xi}:=M_{\xi} / G_{\xi}$. It is known $[13,14]$ that this parametrization uniquely determines the points $(\bar{T}, \bar{c} ; \bar{l}, \bar{k}) \in M_{\xi} \subset M$, which are invariant with respect to the appropriate loop group $\tilde{G}$ action (2) and (7). The last property makes it possible $[10,13,14,20]$ to define on the phase space $\bar{M}_{\xi}$ the reduced nondegenerate symplectic structure on the phase space $\bar{M}_{\xi}$ by means of the appropriate symplectic structure on the submanifold $M_{\xi}$. Let us consider the point $(\bar{T}, \bar{c} ; \bar{l}, \bar{k}) \in M_{\xi}$, where the elements $\bar{T} \in \tilde{\mathcal{G}}, \bar{k} \in \mathbb{C}$, according to the definition (15), satisfy the differential expressions:

$$
[\bar{T}, \bar{l}]-\bar{k}_{x}=0, \quad \bar{k}_{x}=0,
$$

for all $x \in \mathbb{S}^{1}$. Consider now a Hamiltonian vector field $-\bar{k} \mathrm{~d} / \mathrm{d} \tau, \tau \in \mathbb{C}$, on the submanifold $M_{\xi}$, generated by the element $X=\bar{l} \in \tilde{\mathcal{G}}^{*}$ owing to the expressions

$$
\begin{aligned}
& -\bar{k} \bar{T}_{\tau}=[\bar{l}, \bar{T}]=-[\bar{T}, \bar{l}]=-\bar{k} \bar{T}_{x}, \\
& -\bar{k} \bar{l}_{\tau}=\bar{k} \bar{l}_{x} .
\end{aligned}
$$

From (17) it follows that the equality $\frac{\mathrm{d}}{\mathrm{d} \tau}=\frac{\mathrm{d}}{\mathrm{d} x}$ holds on the reduced phase space $\bar{M}_{\xi}$. Let us also compute the evolution of the element $\bar{c} \in \mathbb{C}$ with respect to this vector field $\mathrm{d} / \mathrm{d} \tau$ on $\bar{M}_{\xi}$ :

$$
\begin{aligned}
-\bar{k} \bar{c}_{\tau} & =\left(\overline{l_{x}}, \bar{T}\right)_{-1}=-\left(\bar{l}, \bar{T}_{x}\right)_{-1} \\
& =-\left(\bar{l}, \bar{k}^{-1}[\bar{T}, \bar{l}]\right)_{-1} \\
& =\bar{k}^{-1}([\bar{l}, \bar{l}], \bar{T})_{-1}=0,
\end{aligned}
$$

coinciding with the a priori assumed condition $\mathrm{d} \bar{c} / \mathrm{d} x=0$ for any $x \in \mathbb{S}^{1}$.

Define similarly a vector field $\mathrm{d} / \mathrm{d} t, t \in \mathbb{C}$, on the reduced phase space $\bar{M}_{\xi}$, generated by the Lie algebra element $q(\bar{l}) \in \tilde{\mathcal{G}}$, depending on the basis element $\bar{l} \in \tilde{\mathcal{G}}^{*}$ such that

$$
\begin{aligned}
& \bar{T}_{t}=[q(\bar{l}), \bar{T}], \bar{l}_{t}=[q(\bar{l}), \bar{l}]-\overline{k l}_{x}, \\
& \bar{c}_{t}=\left(q_{x}(\bar{l}), \bar{T}\right)_{-1}, \bar{k}_{t}=0 .
\end{aligned}
$$

This, in particular, means that the flows $\mathrm{d} / \mathrm{d} t$ and $\mathrm{d} / \mathrm{d} x$ on the reduced phase space $\bar{M}_{\xi}$ possess the countable set $\gamma_{n}(\bar{l}):=\operatorname{tr} \bar{T}^{n}(\bar{l}), \quad n \in \mathbb{Z}$, of conservation lows, where by definition, the element $\bar{T}(\bar{l}) \in \tilde{\mathcal{G}}$ satisfies for a given element $\bar{l} \in \tilde{\mathcal{G}}^{*}$ the determining equation

$$
-\bar{k} \bar{T}_{x}(\bar{l})=[\bar{l}, \bar{T}(\bar{l})]
$$

for all $x \in \mathbb{S}^{1}$. From the Equation (20) one easily finds that upon the reduced phase space $\bar{M}_{\xi}$

$$
\begin{aligned}
& \bar{c}_{t}=\left(q(\bar{l})_{x}, \bar{T}\right)_{-1}=\bar{k}^{-1}\left([q(\bar{l}), \bar{l}]-\overline{l_{t}}, \bar{T}\right)_{-1} \\
& =\bar{k}^{-1}([q(\bar{l}), \bar{l}], \bar{T})_{-1}-\bar{k}^{-1}(\bar{l}, \bar{T}) \\
& =\bar{k}^{-1}([\bar{T}, q(\bar{l})], \bar{l})_{-1}-\bar{k}^{-1}\left(\bar{l}_{t}, \bar{T}\right)_{-1} \\
& =-\bar{k}^{-1}\left(\bar{l}, \bar{T}_{t}\right)_{-1}-\bar{k}^{-1}(\bar{l}, \bar{T})_{-1} \\
& =-\bar{k}^{-1} \frac{\mathrm{d}}{\mathrm{d} t}(\bar{l}, \bar{T})_{-1} .
\end{aligned}
$$

Thus, from the $t$-evolution (21) of the parameter $\bar{c} \in \mathbb{C}$ one finds that the constraint

$$
\bar{c}=-\bar{k}^{-1}(\bar{l}, \bar{T})_{-1}
$$

holds on the reduced phase space $\bar{M}_{\xi}$ subject to the vector field $\mathrm{d} / \mathrm{d} t$ generated by the element $q(\bar{l}) \in \tilde{\mathcal{G}}$. Moreover, as it is easy to observe, these two vector fields $\mathrm{d} / \mathrm{d} \tau$ and $\mathrm{d} / \mathrm{d} t$ on the reduced phase space $\bar{M}_{\xi}$ commute:

$$
[\mathrm{d} / \mathrm{d} t, \mathrm{~d} / \mathrm{d} \tau]=0 .
$$

This is very promising, since the condition (23) results in a differential relationships on the components of the reduced matrix $\bar{l} \in \tilde{\mathcal{G}}^{*}$, for which the related evolution equation

$$
\bar{F}_{x}=\overline{l F},
$$

and the differential Equation

$$
\bar{F}_{t}=q(\bar{l}) \bar{F}
$$

for the matrix $F \in \tilde{G}$ are compatible. These Equations (24) and (25) realize the well-known $[1,4-6,10,12]$ generalized Lax spectral problem, allowing to integrate the above differential relationships by means of either the inverse scattering or the spectral transform methods $[1,4,5,21]$ and algebraic geometry methods $[4,5]$, or their modern generalizations [6].

To make this aim more constructive, it is necessary to describe the evolution of the vector field $\mathrm{d} / \mathrm{d} t$ on the reduced phase space $\bar{M}_{\xi}$ in more detail subject to its dependence on the phase space element $\bar{l} \in \tilde{\mathcal{G}}^{*}$. Taking into account that the vector fields $\mathrm{d} / \mathrm{d} t$ and $\mathrm{d} / \mathrm{d} x$ satisfy the commutation condition (23) on the reduced manifold $M_{\xi}$, we will apply Marsden-Weinstein reduction theory to our symplectic manifold $M$ with the fixed value of the moment mapping $\xi=0$ for computing the Poisson bracket

$$
\left\{(\bar{T}, X)_{-1},(\bar{T}, Y)_{-1}\right\}_{\xi}
$$

of the functions $(\bar{T}, X)$ and $(\bar{T}, Y)$ on the reduced phase space $\bar{M}_{\xi}$ for arbitrary $X, Y \in \tilde{\mathcal{G}}^{*}$. It can be shown $[10,20,22]$ that this Poisson bracket on $\bar{M}_{\xi}$ in general is 


$$
\left\{(\bar{T}, X)_{-1},(\bar{T}, Y)_{-1}\right\}_{\xi}=\left.\left\{(\bar{T}, X)_{-1},(\bar{T}, Y)_{-1}\right\}\right|_{\bar{M}_{\xi}}-\left.\left(\xi,\left[V_{X}, V_{Y}\right]\right)_{-1}\right|_{\bar{M}_{\xi}},
$$

where, by definition, the mappings $V_{X}, V_{Y}: \bar{M}_{\xi} \rightarrow \tilde{\mathcal{G}}$ denote the solutions to the relationship

$$
\begin{aligned}
& \left(\xi,\left[Z, V_{X}\right]\right)_{-1}=K_{Z}(T, X)_{-1}, \\
& \left(\xi,\left[Z, V_{Y}\right]\right)_{-1}=K_{Z}(T, Y)_{-1},
\end{aligned}
$$

which holds for all $Z \in \tilde{\mathcal{G}}$. The functions $(\bar{T}, X)$, $(\bar{T}, Y)_{-1} \in \mathcal{D}\left(\bar{M}_{\xi}\right)$ should be extended to the whole phase space $M$ in such a way that their restrictions on the submanifold $M_{\xi} \subset M$ are $\tilde{G}$-invariant.

To apply the Marsden-Weinstein reduction, we will take into account that, by definition, there exists a group element $g(l) \in \tilde{G}$ such that for arbitrarily chosen $l \in \tilde{G}$ the expression

$$
l=g(l) \bar{l}(l) g(l)^{-1}-\bar{k} g_{x}(l) g(l)^{-1}
$$

holds and satisfies the normalization condition $g(\bar{l})=$ $\mathrm{Id} \in \tilde{G}$. By considering the function

$$
f_{X}:=\left(T, g(l) X g(l)^{-1}\right)_{-1},
$$

one can observe that $\left.f_{X}\right|_{\bar{M}_{\xi}}=(\bar{T}, X)_{-1}$ and, by construction, it is $\tilde{G}$-invariant. This means that $f_{X} \in \mathcal{D}\left(M_{\xi}\right)$ for any $l \in \tilde{\mathcal{G}}^{*}$. In fact, for any $a \in \tilde{G}_{\xi}=\tilde{G}$

$$
\begin{aligned}
a \circ f_{X} & :=\left(a \cdot T, g(a \circ l) X g(a \circ l)^{-1}\right)_{-1} \\
& =\left(a T a^{-1}, a g(l) X g(l)^{-1} \cdot a^{-1}\right) \\
& =\left(T, g(l) X g(l)^{-1}\right)_{-1}=f_{X},
\end{aligned}
$$

where we made use of the property $g(a \circ l)=a g(l)$, $l \in \tilde{\mathcal{G}}^{*}$. This holds owing to the definitions (29) and (7):

$$
\begin{aligned}
& a \circ l=a l a^{-1}-\bar{k} a_{x} a^{-1} \\
& =a\left(g(l) \overline{l g}(l)^{-1}-\bar{k} g_{x}(l) g(l)^{-1}\right) a^{-1}-\bar{k} a_{x} a^{-1} \\
& =a g(l) \bar{l}(a g(l))^{-1}-\bar{k} a g_{x}(l) g(l)^{-1} a^{-1}-\bar{k} a_{x} a^{-1} \\
& =a g(l) \bar{l}(a g(l))^{-1}-\bar{k}(a g(l))_{x}(a g(l))^{-1} \\
& =g(a \circ l) \overline{l g}(a \circ l)^{-1}-\overline{k g}_{x}(a \circ l) g(a \circ l)^{-1},
\end{aligned}
$$

giving rise to relationship $g(a \circ l)=a g(l)$ for any $a \in \tilde{G}_{\xi}$ and $l \in \tilde{\mathcal{G}}^{*}$.

Returning to the Poisson bracket (27), we can replace the functions $(\bar{T}, X)_{-1}$ and $(\bar{T}, Y)_{-1} \in \mathcal{D}\left(\bar{M}_{\xi}\right)$ with their $\tilde{G}_{\xi}$-invariant extensions $f_{X} \in \mathcal{D}\left(M_{\xi}\right)$. Before calculating the corresponding Poisson bracket

$$
\begin{aligned}
\left\{\bar{f}_{X}, \bar{f}_{Y}\right\}_{\xi} & =\left.\left\{\bar{f}_{X}, \bar{f}_{Y}\right\}\right|_{\bar{M}_{\xi}}-\left(\xi,\left[V_{X}, V_{Y}\right]\right)_{-1} \\
& =\left.\left\{f_{X}, f_{Y}\right\}\right|_{\bar{M}_{\xi}}-\left.K_{V_{X}} f_{Y}\right|_{\bar{M}_{\xi}},
\end{aligned}
$$

where $K_{V_{X}}: M \rightarrow T(M)$ is the vector field generated on $M$ by the element $V_{X} \in \tilde{\mathcal{G}}$, we need to calculate the action $K_{Z} f_{Y}$ for any element $Z \in \tilde{\mathcal{G}}$. Just as with the calculations from [22], one finds that on the submanifold $M_{\xi}$

$$
\begin{aligned}
K_{Z} f_{Y} & =\left.\frac{\mathrm{d}}{\mathrm{d} \varepsilon}\left(\exp (\varepsilon Z) T \exp (-\varepsilon Z) g(\exp (\varepsilon Z) \circ l) Y g(\exp (\varepsilon Z) \circ l)^{-1}\right)_{-1}\right|_{\varepsilon=0} \\
& =\left(T, g(l)\left[g(l)^{-1} g^{\prime}(l)\left([Z, l]-\bar{k} Z_{x}\right)-g(l)^{-1} Z g(l), Y\right] g(l)^{-1}\right)_{-1}
\end{aligned}
$$

Thus, on the reduced phase space $\bar{M}_{\xi}$ the general expression (34) implies

$$
\left.K_{V_{X}} f_{Y}\right|_{\bar{M}_{\xi}}=\left(\bar{T},\left[g^{\prime}(\bar{l}) \cdot\left(\left[V_{x}, \bar{l}\right]-\bar{k} \frac{\mathrm{d}}{\mathrm{d} x} V_{x}\right)-V_{X}, Y\right]\right)_{-1} .
$$

Therefore, the Poisson bracket (33), in view of the relationships $\left\{f_{X}, f_{Y}\right\}=-\omega^{(2)}\left(K_{V_{X}}, K_{V_{Y}}\right)$ and (35), becomes

$$
\begin{aligned}
\{(\bar{T}, X),(\bar{T}, Y)\}_{\xi} & =\left(\bar{T},\left[g^{\prime}(\bar{l})(Y), X\right]+\left[Y, g^{\prime}(\bar{l})(X)\right]\right)_{-1}-\left(\bar{T},\left[g^{\prime}(\bar{l})\left(\left[V_{X}, \bar{l}\right]-\bar{k} \frac{\mathrm{d}}{\mathrm{d} X} V_{X}\right)-V_{X}, Y\right]\right)_{-1} \\
& =\left(\bar{T},\left[g^{\prime}(\bar{l})(Y), X\right]+\left[Y, g^{\prime}(\bar{l})(X)\right]\right)_{-1},
\end{aligned}
$$

where we take into account that owing to (28) and (35), the expression 


$$
\begin{aligned}
& \left(\bar{T},\left[g^{\prime}(\bar{l})\left(\left[V_{X}, \bar{l}\right]-\bar{k} \frac{\mathrm{d}}{\mathrm{d} x} V_{X}\right)-V_{X}, Y\right]\right)_{-1}=K_{V_{X}} f_{Y} \\
& =\left.\left(\xi,\left[K_{V_{X}}, V_{Y}\right]\right)_{-1}\right|_{\xi=0}=0 .
\end{aligned}
$$

Now one can rewrite the Poisson bracket (36) as

$$
\{(\bar{T}, X),(\bar{T}, Y)\}_{\xi}=\left(\bar{T},[X, Y]_{D}\right)_{-1},
$$

where, by definition, we have introduced the classical $D$-matrix structure in the Lie algebra $\tilde{\mathcal{G}}^{*}$ :

$$
[X, Y]_{D}:=[D(X), Y]+[X, D(Y)],
$$

where $X, Y \in \tilde{\mathcal{G}}^{*}$ and the linear homomorphism $D: \tilde{\mathcal{G}}^{*} \rightarrow \tilde{\mathcal{G}}^{*}$ is defined as

$$
D(X):=-g^{\prime}(\bar{l})(X) .
$$

The mapping (39) should satisfy [23] the well-known condition

$$
\begin{aligned}
& \left(\bar{T},\left[X,[D(Y), D(Z)]-D[Y, Z]_{D}\right]\right)_{-1} \\
& +(\bar{T},[X,\{(\bar{T}, Y),(\bar{T}, Z)\}])+\text { cycles }=0
\end{aligned}
$$

for any $X, Y \in \tilde{\mathcal{G}}^{*}$ and $Z \in \tilde{\mathcal{G}}$.

Now it is useful to recall that the mapping $g: \tilde{\mathcal{G}}^{*} \rightarrow \tilde{G}$ satisfies the relationship (29), which implies [24] the following differential expression

$$
\left[g^{\prime}(\bar{l})(X), \bar{l}\right]-\bar{k} \frac{\mathrm{d}}{\mathrm{d} x} g^{\prime}(\bar{l})(X)=X
$$

for any $X \in \tilde{\mathcal{G}}^{*}$, where $g^{\prime}(\bar{l}): \tilde{\mathcal{G}}^{*} \rightarrow \tilde{\mathcal{G}}^{*}$ is the derivative mapping depending on the chosen reduction $\tilde{\mathcal{G}} \ni l \rightarrow \bar{l} \in \tilde{\mathcal{G}}^{*}$.

The mapping (39) satisfies an additional relationship, which can be obtained from the group $\tilde{G}$-action on the element $\bar{T}(\bar{l}) \in \tilde{\mathcal{G}}$ :

$$
T(l)=g(l) \bar{T}(\bar{l}) g(l)^{-1},
$$

following naturally from (29). Differentiation of (42) with respect to $l \in \tilde{\mathcal{G}}^{*}$ at the point $l=\bar{l}$, gives rise to

$$
T^{\prime}(\bar{l})(X)=\left[g^{\prime}(\bar{l})(X), \bar{T}(\bar{l})\right]
$$

for an arbitrary $X \in \tilde{\mathcal{G}}^{*}$. Moreover, since the matrix (42) satisfies the relationship (20), its differentiation with respect to $\bar{l} \in \tilde{\mathcal{G}}^{*}$ yields the differential expression:

$$
\bar{k} \frac{\mathrm{d}}{\mathrm{d} x} T^{\prime}(\bar{l})(Y)+\left[\bar{l}, T^{\prime}(\bar{l})(Y)\right]=[\bar{T}(\bar{l}), Y],
$$

which holds for any $Y \in \tilde{\mathcal{G}}^{*}$. The above results can be formulated as the following proposition.

Proposition 1 The Poisson bracket (26) on the reduced phase space $\bar{M}_{\xi}$ represented as a $D$-structure (37) on the linear space $\tilde{\mathcal{G}}^{*}$, naturally generated by the gauge transformation (29), which reduces the arbitrary element $l \in \tilde{\mathcal{G}}^{*}$ to the element $\bar{l} \in \tilde{\mathcal{G}}^{*}$, is uniquely defined on $\bar{M}_{\xi}$.

As a consequence of representation (37) we find that there exists an another infinite hierarchy of mutually commuting functionals with respect to the Poisson bracket on the phase space $\bar{M}_{\xi}$. The latter follows from the tensor form of the Poisson bracket (26) in the space $\tilde{\mathcal{G}} \otimes \tilde{\mathcal{G}}:$

$$
\begin{aligned}
& \{\bar{T}(\bar{l})(\lambda) \otimes, \widehat{T}(\bar{l})(\mu)\}_{\xi} \\
& =[D(\lambda, \mu), \bar{T}(\bar{l})(\lambda) \otimes \mathbb{I}+\mathbb{I} \otimes \bar{T}(\bar{l})(\mu)]
\end{aligned}
$$

which holds for arbitrary $\lambda, \mu \in \mathbb{C}$ and where $D(\lambda, \mu): \tilde{\mathcal{G}}^{*} \rightarrow \tilde{\mathcal{G}}^{*}$ denotes the tensor form of the $D$ structure $D: \tilde{\mathcal{G}}^{*} \rightarrow \tilde{\mathcal{G}}^{*}$. The trace operation in (45) causes the Poisson bracket to vanish on the phase space $\bar{M}_{\xi}$ for the functionals $\operatorname{tr} \bar{T}(\bar{l})(\lambda)$ and $\operatorname{tr} \bar{T}(\bar{l})(\mu)$ for arbitrary $\lambda, \mu \in \mathbb{C}$.

\section{Monodromy, $R$-Structure and Lie-Poisson Brackets}

Next we analyze possible forms of the $D$-mapping (39) as a function on the reduced phase space $\bar{M}_{\xi}$. Since $\bar{k} \in \mathbb{C}$ is constant, its value for convenience is set at $\bar{k}=-1$. Thus, taking into account the definition (39), the determining $D$-structure Equation (41) takes the form:

$$
[D(\bar{l})(Y), \bar{l}]+\frac{\mathrm{d}}{\mathrm{d} x} D(\bar{l})(Y)+Y=0
$$

for any element $Y \in \tilde{\mathcal{G}}^{*}$.

Let us consider the linear matrix Equation

$$
\bar{F}_{x}(x, s ; \lambda)=\bar{l}(x ; \lambda) \bar{F}(x, s ; \lambda),
$$

where $\bar{l}(x ; \lambda) \in \tilde{\mathcal{G}}^{*}, \bar{F} \in \tilde{G}$, with Cauchy data at a point $x=s \in \mathbb{S}^{1}$ :

$$
\left.\bar{F}(x, s ; \lambda)\right|_{x=s}=\mathbb{I} .
$$

The corresponding normalized monodromy matrix

$$
\bar{T}(x ; \lambda):=\bar{F}(x+2 \pi, x ; \lambda)-v^{-1} \operatorname{It} \bar{F}(x+2 \pi, x ; \lambda),
$$

for $x \in \mathbb{S}^{1}$ and arbitrary $\lambda \in \mathbb{C}$ satisfies

$$
\bar{T}_{x}-[\bar{T}, \bar{l}]=0,
$$

exactly coinciding with (20). Thus, if by the co-adjoint transformation (7) this chosen matrix $l \in \tilde{\mathcal{G}}^{*}$ is transformed into the matrix $\bar{l} \in \tilde{\mathcal{G}}^{*}$, then the corresponding monodromy matrix of (24) transforms into the monodromy matrix of (47), which satisfies (50).

In view of the relationships (47), (48) and (50), one can recalculate the Poisson bracket (37) as 


$$
\bar{T}(\bar{l})(z ; \lambda)=\bar{T}(z ; \lambda)
$$

for arbitrary $z \in \mathbb{S}^{1}$ and $\lambda \in \mathbb{C}^{1}$. It yields the following tensor expression for the reduced phase space $\bar{M}_{\xi}$ :

$$
\begin{aligned}
& \{\bar{T}(\bar{l})(z ; \lambda) \otimes \bar{T}(\bar{l})(z ; \mu)\}_{\xi} \\
& =\int_{z}^{z+2 \pi} \mathrm{d} x \int_{z}^{z+2 \pi} \mathrm{d} y\{F(z+2 \pi, x ; \lambda) \bar{l}(x ; \lambda) F(x, z ; \lambda) \otimes F(z+2 \pi, y ; \mu) \bar{l}(y ; \mu) F(y, z ; \mu)\}_{\xi} \\
& =\int_{z}^{z+2 \pi} \mathrm{d} x \int_{z}^{z+2 \pi} \mathrm{d} y\{(F(z+2 \pi, x ; \lambda) \otimes \mathbb{I})(\bar{l}(x ; \lambda) \otimes \mathbb{I}) F(x, z ; \lambda) \otimes \mathbb{I}, \mathbb{I} \otimes F(z+2 \pi, y ; \mu)(\mathbb{I} \otimes \bar{l}(y ; \mu))(\mathbb{I} \otimes F(y, z ; \mu))\}_{\xi} \\
& =\int_{z}^{z+2 \pi} \mathrm{d} x \int_{z}^{z+2 \pi} \mathrm{d} y F(z+2 \pi, x ; \lambda) \otimes F(z+2 \pi, y ; \mu)\{\bar{l}(x ; \lambda) \otimes \bar{l}(y ; \mu)\}_{\xi} F(x, z ; \lambda) \otimes F(y, z ; \mu) \\
& =\int_{z}^{++2 \pi} \mathrm{d} x \int_{z}^{z+2 \pi} \mathrm{d} y F(z+2 \pi, x ; \lambda) \otimes F(z+2 \pi, y ; \mu) \bar{\omega}(\lambda, \mu ; x, y) F(x, z ; \lambda) \otimes F(y, z ; \mu),
\end{aligned}
$$

where $z \in \mathbb{S}^{1}, \lambda, \mu \in \mathbb{C}$ and, by definition,

$$
\{\bar{l}(x ; \lambda) \underset{\otimes}{\otimes} \bar{I}(y ; \mu)\}_{\xi}:=\bar{\omega}(\lambda, \mu ; x, y)=\sum_{i, k=0}^{N} \bar{\omega}_{i k}(\lambda, \mu ; x, y) \partial_{x}^{i} \partial_{y}^{k} \delta(x-y) .
$$

The local functional matrices

$$
\bar{\omega}_{i k}(\lambda, \mu ; x, y) \in \tilde{\mathcal{G}}^{*} \otimes \tilde{\mathcal{G}}^{*}
$$

satisfy the antisymmetry property:

$$
\begin{aligned}
& P \bar{\omega}_{i k}(\lambda, \mu ; x, y) P=-\bar{\omega}_{k i}(\mu, \lambda ; x, y) \\
& \qquad \bar{T}(z ; \lambda) \stackrel{\otimes}{,} \bar{T}(z ; \mu)\}_{\xi}=\int_{z}^{z+2 \pi} \mathrm{d} x \bar{F}(z+ \\
& \text { the matrix } \bar{\Omega}(\lambda, \mu ; x) \in \tilde{\mathcal{G}}^{*} \otimes \tilde{\mathcal{G}}^{*} \\
& \text { C, } x \in \mathbb{S}^{1}, \text { depends only on } \bar{l} \in \mathcal{G}^{*} \text {. } \\
& \{\bar{T}(z ; \lambda) \otimes \bar{T}(z ; \mu)\}_{\xi} \\
& =\mathcal{R}(\lambda, \mu ; z) \bar{T}(z ; \lambda) \otimes \bar{T}(z ; \mu) \\
& -\bar{T}(z ; \lambda) \otimes \bar{T}(z ; \mu) \mathcal{R}(\lambda, \mu ; z),
\end{aligned}
$$$$
\{\bar{T}(z ; \lambda) \stackrel{\otimes}{,} \bar{T}(z ; \mu)\}_{\xi}=\int_{z}^{z+2 \pi} \mathrm{d} x \bar{F}(z+2 \pi, x ; \lambda) \otimes \bar{F}(z+2 \pi, x ; \mu) \bar{\Omega}(\lambda, \mu ; x) \bar{F}(x, z ; \lambda) \otimes \bar{F}(x, z ; \mu),
$$

for all $i, k=\overline{1, N}, \quad x, y \in \mathbb{S}^{1}, \quad \lambda, \mu \in \mathbb{C}$ and the permutation operator $P: \tilde{\mathcal{G}}^{*} \otimes \tilde{\mathcal{G}}^{*}$, acts as $P A \otimes B P:=B \otimes A$ for any $A, B \in \tilde{\mathcal{G}}^{*}$. Just as in the calculation from $[1,25,26]$ one obtains from (53) that where the matrix $\bar{\Omega}(\lambda, \mu ; x) \in \tilde{\mathcal{G}}^{*} \otimes \tilde{\mathcal{G}}^{*} \quad$ for $\quad$ all $\lambda, \mu \in \mathbb{C}, x \in \mathbb{S}^{1}$, depends only on $\bar{l} \in \mathcal{G}^{*}$. The expression (55) allows the very compact representation

if the tensor $\mathcal{R}$-matrix $\mathcal{R} \in \tilde{\mathcal{G}} \otimes \tilde{\mathcal{G}}^{*}$ satisfies for $x \in \mathbb{S}^{1}$ and $\lambda, \mu \in \mathbb{C}$ the differential relationship

$$
\begin{aligned}
& \frac{\mathrm{d}}{\mathrm{d} x} \mathcal{R}(\lambda, \mu ; x) \\
& +[\mathcal{R}(\lambda, \mu ; x), l(x ; \lambda) \otimes \mathbb{I}+\mathbb{I} \otimes l(x ; \mu)] \\
& =\Omega(\lambda, \mu ; x) .
\end{aligned}
$$

If we define the mapping $R: \tilde{\mathcal{G}} \rightarrow \tilde{\mathcal{G}}$ as

$$
R(Y):=\operatorname{res} \int_{\mu=0}^{2 \pi} \mathrm{d} y \mathcal{R}(\lambda, \mu ; y) \delta(x-y) Y(y ; \mu)
$$

for any $Y \in \tilde{\mathcal{G}}^{*}$, then the relationship (57) can be easily presented in the following operator form:

$$
-\left(X, \frac{\mathrm{d} R}{\mathrm{~d} x}(Y)\right)_{-1}+\left(\bar{l},[X, Y]_{R}\right)=(X, R(Y))_{-1},
$$

which holds for any $X, Y \in \tilde{\mathcal{G}}$, where we denoted

$$
[X, Y]_{R}:=\left[-R^{*}(x), Y\right]+[X, R(Y)] .
$$

The result (59) can be used for rewriting the Poisson bracket (56) as

$$
\begin{aligned}
& \left\{(X, \bar{T}(\bar{l}))_{-1},(Y, \bar{T}(\bar{l}))_{-1}\right\}_{\xi} \\
& =\left(\bar{l},\left[\bar{F} X \bar{F}_{2 \pi}, \bar{F} Y \bar{F}_{2 \pi}\right]_{R}\right)_{-1}-\left(\bar{F} X \bar{F}_{2 \pi}, \frac{R}{\mathrm{~d} x}\left(\bar{F} Y \bar{F}_{2 \pi}\right)\right)_{-1} \\
& =\left(\bar{l},[\nabla(X, \bar{T})(\bar{l}), \nabla(Y, \bar{T})(\bar{l})]_{R}\right)_{-1} \\
& -\left(\nabla(X, \bar{T})(\bar{l}), \frac{\mathrm{d}}{\mathrm{d} x} R(\nabla(Y, \bar{T})(\bar{l}))\right)_{-1} \\
& -\left(R^{*}(\nabla(X, \bar{T})(\bar{l})), \frac{\mathrm{d}}{\mathrm{d} x}(\nabla(Y, \bar{T})(\bar{l}))\right)_{-1},
\end{aligned}
$$

where

$$
\bar{F}:=\bar{F}(\bar{l})(x, y ; \lambda), \bar{F}_{2 \pi}:=\bar{F}(\bar{l})(y+2 \pi, x ; \lambda) \in \tilde{G},
$$


$x, y \in \mathbb{S}^{1}, \lambda \in \mathbb{C}$, and we defined the gradients $\nabla(X, \bar{T})(\bar{l})$ and $\nabla(Y, \bar{T})(\bar{l}) \in \tilde{\mathcal{G}}$ in the standard way as

$$
(\nabla f(\bar{l}), Z)_{-1}:=\left.\frac{\mathrm{d}}{\mathrm{d} \varepsilon} f(\bar{l}+\varepsilon Z)\right|_{\varepsilon=0}
$$

for any smooth functional $f \in \mathcal{D}\left(\tilde{\mathcal{G}}^{*}\right)$ and $Z \in \tilde{\mathcal{G}}^{*}$.

It is easy to observe that under the antisymmetry condition $R^{*}=-R$ the right-hand side of (61) equals the Lie-Poisson bracket $[1,2,4,6,10]$ for the functionals $(X, \bar{T})$ and $(Y, \bar{T}) \in \mathcal{D}\left(\tilde{\mathcal{G}}^{*}\right)$. Here the adjoint space $\tilde{\mathcal{G}}^{*}=\tilde{\mathcal{G}}^{*} \oplus \mathbb{C}$ is with respect to a new commutator structure $[\cdot, \cdot]_{R}$ on the centrally extended Lie algebra $\hat{\mathcal{G}}$ : for any $(X, c),(Y, r) \in \hat{\mathcal{G}}$ with commutator

$$
\begin{aligned}
& {[(X, c),(Y, r)]_{R}} \\
& =\left([X, Y]_{R},\left(\frac{\mathrm{d}}{\mathrm{d} x} X, R(Y)\right)_{-1}+\left(\frac{\mathrm{d}}{\mathrm{d} x} R(X), Y\right)_{-1}\right) .
\end{aligned}
$$

In (63) the classical $R$-structure on the Lie algebra $\tilde{\mathcal{G}}[X, Y]_{R}:=[R(X), Y]+[X, R(Y)]$ under some conditions on the mapping $R: \tilde{\mathcal{G}} \rightarrow \tilde{\mathcal{G}}$ can generate on $\tilde{\mathcal{G}}$ a new Lie structure (which it must not).

The above results can be formulated as follows.

Proposition 2 The Marsden-Weinstein reduced canonical Poisson structure on the phase space $\bar{M}$ for the monodromy matrix $\bar{T}(\bar{l}) \in \tilde{\mathcal{G}}$ exactly coincides with the corresponding classical Lie-Poisson AKS-bracket on the centrally extended basis Lie algebra $\hat{\mathcal{G}}$ subject to the $R$-structure (63) when it is antisymmetric.

If the antisymmetry property for the mapping $R: \tilde{\mathcal{G}} \rightarrow \tilde{\mathcal{G}}$ does not hold, the generated Lie-Poisson type bracket on the functional space $\mathcal{D}\left(\tilde{\mathcal{G}}^{*}\right)$ can be, owing to (61), defined as follows: for any $f, g \in \mathcal{D}\left(\tilde{\mathcal{G}}^{*}\right)$ the bracket

$$
\begin{aligned}
\{f(\bar{l}), g(\bar{l})\}_{\xi} & :=\left(\bar{l},[\nabla f(\bar{l}), \nabla g(\bar{l})]_{R}\right)_{-1} \\
& +\left(\frac{\mathrm{d}}{\mathrm{d} x} \nabla f(\bar{l}), R(\nabla g(\bar{l}))\right)_{-1} \\
& +\left(\frac{\mathrm{d}}{\mathrm{d} x}(R \nabla f(\bar{l})), \nabla g(\bar{l})\right)_{-1},
\end{aligned}
$$

where the generalized $R$-structure $[\cdot, \cdot]_{R}$ on $\tilde{\mathcal{G}}$ is given by the expression (60).

\section{5. $\boldsymbol{D}$-Structure and Generalized $\boldsymbol{R}$-Structure}

As stated above, the reduced Poisson bracket on the phase space $\bar{M}_{\xi}$ is

$$
\{(X, \bar{T}),(Y, \bar{T})\}_{\xi}=\left(\bar{T},[X, Y]_{D}\right)_{-1},
$$

where for any $X, Y \in \tilde{\mathcal{G}}$ the corresponding $D$-struc- ture on the Lie algebra $\tilde{\mathcal{G}}$ is defined by the classical expression (38) and the mapping (39). It is natural to assume that there exists a relationship between the $D$-structure $D: \tilde{\mathcal{G}} \rightarrow \tilde{\mathcal{G}}$ and the $R$-structure $R: \tilde{\mathcal{G}} \rightarrow \tilde{\mathcal{G}}$, described above in Section 3.

Assume, for brevity, that the $R$-structure (58) is antisymmetric, that is $R^{*}=-R$. Then it is easy to check that the following algebraic relationship

$$
D(X):=\frac{1}{2} R(\bar{T} X+X \bar{T})
$$

holds for any $X \in \tilde{\mathcal{G}}$. In fact, (56) is equivalent to

$$
\begin{aligned}
\{(\bar{T}, X),(\bar{T}, Y)\}_{\xi} & =(\bar{T} X, R(\bar{T} Y))_{-1} \\
& -(X \bar{T}, R(Y \bar{T}))_{-1} .
\end{aligned}
$$

Now, substituting (66) into (37), one obtains that

$$
\begin{aligned}
\{(\bar{T}, X),(\bar{T}, Y)\}_{\xi} \\
=\frac{1}{2}(\bar{T},[R(\bar{T} X+X \bar{T}), Y]+[X, R(\bar{T} Y+Y \bar{T})])_{-1} \\
=\frac{1}{2}([Y, \bar{T}], R(\bar{T} X))_{-1}+\frac{1}{2}([Y, \bar{T}], R(X \bar{T}))_{-1} \\
+\frac{1}{2}([\bar{T}, X], R(\bar{T} Y))_{-1}+\frac{1}{2}([\bar{T} X, R(Y \bar{T})])_{-1} \\
=\frac{1}{2}(Y \bar{T}, R(\bar{T} X))_{-1}-\frac{1}{2}(\bar{T} Y, R(\bar{T} X))_{-1} \\
+\frac{1}{2}(Y \bar{T}, R(X \bar{T}))_{-1}-\frac{1}{2}(\bar{T} Y, R(X \bar{T}))_{-1} \\
+\frac{1}{2}(\bar{T} X, R(\bar{T} Y))_{-1}-\frac{1}{2}(X \bar{T}, R(\bar{T} X))_{-1} \\
+\frac{1}{2}(\bar{T} X, R(Y \bar{T}))_{-1}-\frac{1}{2}(X \bar{T}, R(Y \bar{T}))_{-1} \\
=(\bar{T} X, R(\bar{T} Y))_{-1}-(X \bar{T}, R(Y \bar{T}))_{-1}
\end{aligned}
$$

which coincides exactly with (67).

It is convenient to rewrite the operator relationship (46) in the tensor form as

$$
(\bar{l} \otimes \mathbb{I}) D-D(\mathbb{I} \otimes \bar{l})-\frac{\mathrm{d}}{\mathrm{d} x} D=\mathbb{I},
$$

where the tensor $D \in \tilde{\mathcal{G}} \otimes \tilde{\mathcal{G}}^{*}$, owing to the action (66), equals

$$
D=\frac{1}{2}(R(\mathbb{I} \otimes \bar{T})+(\mathbb{I} \otimes \bar{T}) R) .
$$

Substituting the expression (70) into the Equation (69) and taking into account the determining Equation (57)

$$
[\bar{l} \otimes \mathbb{I}+\mathbb{I} \otimes \bar{l}, R]-\frac{\mathrm{d}}{\mathrm{d} x} R=\bar{\Omega},
$$

one obtains the relationship for the tensor $\bar{\Omega} \in \tilde{\mathcal{G}} \rightarrow \tilde{\mathcal{G}}^{*}$ : 


$$
\begin{aligned}
2 \mathbb{I} \otimes \mathbb{I}-\bar{\Omega}= & {[R, \mathbb{I} \otimes \overline{T l}]+(\mathbb{I} \otimes \bar{T}) R(\mathbb{I} \otimes \bar{l}) } \\
& -(\mathbb{I} \otimes \bar{l}) R(\mathbb{I} \otimes \bar{T}) .
\end{aligned}
$$

This makes two $R$ - and $D$-structures on the Lie algebra $\tilde{\mathcal{G}}$ compatible. Observe that the $D$-structure (66) is not antisymmetric even though the $R$-structure was assumed to be antisymmetric. Concerning the $D$-structure determining Equation (69) one can anticipate that a study of its solutions would describe a set of nonlinear dynamical systems on the reduced phase space $\bar{M}_{\xi}$ possessing an infinite hierarchy of mutually commuting conservation laws.

\section{Generalized Riemann Systems: Lax Integrability and D-A Structures}

\subsection{Setting the Problem}

Recently, new mathematical approaches based on differential-algebraic [27-31] and differential geometric methods and techniques, were applied in $[8,32,33]$ for studying the Lax integrability of nonlinear differential equations of the Korteweg-de Vries and Riemann type. In particular, many analytical studies [32,34-40] have been devoted to finding the corresponding Lax representations of the infinite Riemann type hydrodynamical hierarchy, suggested recently by M. Pavlov and D. Holm in the form

$$
D_{t}^{N} u=z,
$$

where the differentiation $D_{t}:=\partial / \partial t+u \partial / \partial x, \quad N \in \mathbb{N}$, $(x, t)^{\top} \in \mathbb{R}^{2}$ and $u \in C^{\infty}(\mathbb{R} / 2 \pi \mathbb{Z} ; \mathbb{R})$. It was found that the related dynamical system

$$
D_{t} u_{1}=u_{2}, \cdots, D_{t} u_{j}=u_{j+1}, \cdots, D_{t} u_{N}=0,
$$

defined on a $2 \pi$-periodic infinite-dimensional smooth functional manifold $M_{N} \subset C^{\infty}\left(\mathbb{R} / 2 \pi \mathbb{Z} ; \mathbb{R}^{N}\right)$, possesses [8,37] for an arbitrary integer $N \in \mathbb{N}_{+}$a suitable Lax representation

$$
D_{x} f=l_{N}[u ; \lambda] f, \quad D_{t} f=q_{N}(\lambda) f
$$

with $\lambda \in \mathbb{C}$ being a complex spectral parameter and $f \in L_{\infty}\left(\mathbb{R} ; \mathbb{C}^{N}\right)$ and matrices

$$
l_{N}[u ; \lambda], q_{N}(\lambda) \in \operatorname{End} \mathbb{C}^{2} .
$$

Here, by definition, $u_{1}:=u \in C^{\infty}\left(\mathbb{R}^{2} ; \mathbb{R}\right)$ and the differentiations

$$
D_{t}:=\partial / \partial t+u_{1} D_{x}, D_{x}:=\partial / \partial x
$$

satisfy on the manifold $M_{N}$ the following commutation relationship:

$$
\left[D_{x}, D_{t}\right]=u_{1, x} D_{x} .
$$

In particular, for $N \in \mathbb{N}$ the following result $[8,41]$ was recently obtained.

Proposition 3 The Lax representation for the generalized Riemann type hydrodynamical system

$$
D_{t} u_{1}=u_{2}, D_{t} u_{2}=u_{3}, \cdots, D_{t} u_{N-1}=u_{N}, D_{t} u_{N}=0
$$

is given for any arbitrary $N \in \mathbb{N}$ by a set of linear compatibility equations (see Equation (79)),

where $f \in C^{\infty}\left(\mathbb{R}^{2} ; \mathbb{C}^{N}\right)$ and $\lambda \in \mathbb{C}$ is an arbitrary complex parameter. Moreover, the relationships (79) realize a linear matrix representation of the commutator condition (77).

In our work we study the complete integrability of a new dispersive Riemann type hydrodynamic flow

$$
D_{t}^{N-1} u=\bar{z}_{x}^{2}, \quad D_{t} \bar{z}=0
$$

on a $2 \pi$-periodic functional manifold

$$
\bar{M}_{N} \subset C^{\infty}\left(\mathbb{R} / 2 \pi \mathbb{Z} ; \mathbb{R}^{N}\right),
$$

where $N \in \mathbb{N}$ is an arbitrary natural number, the vector

$$
\left(u, D_{t} u, D_{t}^{2} u, \cdots, D_{t}^{N-1} u, \bar{z}\right)^{\top} \in \bar{M}_{N},
$$

the differentiations

$$
D_{x}:=\partial / \partial x, \quad D_{t}:=\partial / \partial t+u \partial / \partial x
$$

$$
\begin{aligned}
D_{t} f & =\left(\begin{array}{ccccc}
0 & 0 & 0 & 0 & 0 \\
-\lambda & 0 & 0 & 0 & 0 \\
0 & -\lambda & \ddots & \ldots & 0 \\
0 & 0 & \ddots & 0 & 0 \\
0 & 0 & 0 & -\lambda & 0
\end{array}\right) f, \\
D_{x} f & =\left(\begin{array}{ccccc}
\lambda u_{N-1, x} & u_{N, x} & 0 & \ldots & 0 \\
0 & \lambda u_{N-1, x} & 2 u_{N, x} & \ddots & \ldots \\
\ldots & \ddots & \ddots & \ddots & 0 \\
0 & \ldots & 0 & \lambda u_{N-1, x} & (N-1) u_{N, x} \\
-N \lambda^{N} & -\lambda^{N-1} N u_{1, x} & \cdots & -\lambda^{2} N u_{N-2, x} & \lambda(1-N) u_{N-1, x}
\end{array}\right) f,
\end{aligned}
$$


satisfy as above the Lie-algebraic commutator relationship (77) and $t \in \mathbb{R}$ is an evolution parameter. This system can be considered as a slight generalization of the dispersive Riemann hydrodynamic system (73), extensively studied by means of different mathematical tools in $[8,9,32,35,37,41]$. For the case $N=2$ it is well known $[10,12]$ that the system $(80)$ is a smooth Lax integrable bi-Hamiltonian flow on the $2 \pi$-periodic functional manifold $\bar{M}_{2}$, whose Lax representation is given by the compatible linear system

$$
\begin{aligned}
& D_{x} f=\left(\begin{array}{cc}
\bar{z}_{x} & 0 \\
-\lambda\left(u+u_{x} / \bar{z}_{x}\right) & -\bar{z}_{x x} / \bar{z}_{x}
\end{array}\right) f, \\
& D_{t} f=\left(\begin{array}{cc}
0 & 0 \\
-\lambda \bar{z}_{x} & u_{x}
\end{array}\right) f,
\end{aligned}
$$

where $f \in C^{\infty}\left(\mathbb{R}^{2} ; \mathbb{R}^{2}\right)$ and $\lambda \in \mathbb{R}$ is an arbitrary spectral parameter.

For $N=3$ the dynamical system (80) is equivalent to that on a $2 \pi$-periodic functional manifold

$$
\bar{M}_{3} \subset C^{\infty}\left(\mathbb{R} / 2 \pi \mathbb{Z} ; \mathbb{R}^{3}\right)
$$

for a vector $(u, v, \bar{z})^{\top} \in \bar{M}_{3}$ :

$$
D_{t} u=v, D_{t} v=\bar{z}_{x}^{2}, D_{t} \bar{z}=0 .
$$

This can be easily rewritten by means of the change of variables $z:=\bar{z}_{x}^{2}$ as that on a $2 \pi$-periodic functional manifold

$$
M_{3} \subset C^{\infty}\left(\mathbb{R} / 2 \pi \mathbb{Z} ; \mathbb{R}^{3}\right)
$$

for a vector $(u, v, z)^{\top} \in M_{3}$

$$
D_{t} u=v, \quad D_{t} v=z, \quad D_{t} z=-2 z u_{x},
$$

or in the form of the flow

$$
\left(\begin{array}{l}
\mathrm{d} u / \mathrm{d} t \\
\mathrm{~d} v / \mathrm{d} t \\
\mathrm{~d} z / \mathrm{d} t
\end{array}\right)=K[u, v, z]:=\left(\begin{array}{c}
v-u u_{x} \\
z-u v_{x} \\
-2 u_{x} z-u z_{x}
\end{array}\right),
$$

defining a standard smooth dynamical system on the infinite-dimensional functional manifold $M_{3}$, where $K: M_{3} \rightarrow T\left(M_{3}\right)$ is the corresponding vector field on $M_{3}$. We succeeded in proving the following result based on symplectic gradient-holonomic and differential algebraic tools

Proposition 4 The Riemann type hydrodynamic flow (97) is a bi-Hamiltonian dynamical system on the functional manifold $M_{3}$ with respect to two compatible Poissonian structures $\vartheta, \eta: T^{*}\left(M_{3}\right) \rightarrow T\left(M_{3}\right)$

$$
\begin{aligned}
\vartheta & :=\left(\begin{array}{ccc}
0 & 1 & 0 \\
-1 & 0 & 0 \\
0 & 0 & 2 z^{1 / 2} D_{x} z^{1 / 2}
\end{array}\right), \\
\eta & :=\left(\begin{array}{ccc}
\partial^{-1} & u_{x} \partial^{-1} & 0 \\
\partial^{-1} u_{x} & v_{x} \partial^{-1}+\partial^{-1} v_{x} & \partial^{-1} z_{x}-2 z \\
0 & z_{x} \partial^{-1}+2 z & 0
\end{array}\right),
\end{aligned}
$$

possessing an infinite hierarchy of mutually commuting conservation laws and a non-autonomous Lax representation of the form (see Equation (86)).

where $\lambda \in \mathbb{R}$ is an arbitrary spectral parameter and $f \in C^{\infty}\left(\mathbb{R}^{2} ; \mathbb{R}^{3}\right)$.

We demonstrate the effectiveness of the devised differential-algebraic tools and methods by means of application to the very interesting [42-47] nonlinear Ostrovsky-Vakhnenko hydrodynamic equation

$$
D_{x}\left(D_{t} u+D_{x}^{-1} u\right)=0
$$

on the $2 \pi$-periodic functional manifold

$$
M \subset C^{\infty}(\mathbb{R} / 2 \pi \mathbb{Z} ; \mathbb{R})
$$

subject to which the following proposition is proved.

Proposition 5 The Ostrovsky-Vakhnenko dynamical system (79) allows the standard differential Lax representation and defines on the functional manifold $M$ an integrable bi-Hamiltonian flow with two compatible Poisson structures. In particular, this dynamical system possesses an infinite hierarchy of mutually commuting nonlocal conservation laws.

In particular, we construct by means of the differential-algebraic tools a differential Lax representation, coinciding with that found in [43], and given in the equivalent matrix Zakharov-Shabat form as

$$
D_{t} h=\hat{q}[u ; \mu] h, D_{x} h=\hat{l}[u ; \mu] h,
$$

where matrices (see Equation (89))

$$
\begin{aligned}
D_{t} f & =\left(\begin{array}{ccc}
0 & 0 & 0 \\
-\lambda & 0 & 0 \\
0 & -\lambda z_{x} & u_{x}
\end{array}\right) f, \\
D_{x} f & =\left(\begin{array}{ccc}
\lambda^{2} u \sqrt{z} & \lambda v \sqrt{z} & z \\
-\lambda^{3} t u \sqrt{z} & -\lambda^{2} t v \sqrt{z} & -\lambda t z \\
\lambda^{4}\left(t u v-u^{2}\right)-\lambda^{2} u_{x} / \sqrt{z} & -\lambda v_{x} / \sqrt{z}+\lambda^{3}\left(t v^{2}-u v\right) & \lambda^{2} \sqrt{z}(u-t v)-z_{x} / 2 z
\end{array}\right) f
\end{aligned}
$$




$$
\begin{aligned}
& \hat{q}[u ; \mu]:=\left(\begin{array}{ccc}
u_{x} & 0 & 1 / \mu \\
-1 / 3 & 0 & 0 \\
0 & -1 / 3 & -u_{x}
\end{array}\right), \\
& \hat{\imath}[u ; \mu]:=\left(\begin{array}{ccc}
0 & 1 & 0 \\
0 & 0 & 1 \\
-\mu \bar{u} & 0 & 0
\end{array}\right),
\end{aligned}
$$

$h \in C^{2}\left(\mathbb{R}^{2} ; \mathbb{C}^{3}\right)$ and $\mu \in \mathbb{C}$ is an arbitrary spectral parameter. We also find a related pair of compatible polynomial Poissonian structures

$$
\begin{aligned}
& \vartheta, \eta: T^{*}(M) \rightarrow T(M) \\
& \vartheta=\partial, \\
& \eta=\partial^{-1} \bar{u} \partial^{-3} \bar{u} \partial^{-1}+4 \partial^{-2} \bar{u} \partial^{-1} \bar{u} \partial^{-2} \\
& +2\left(\partial^{-2} \bar{u} \partial^{-2} \bar{u} \partial^{-1}+\partial^{-1} \bar{u} \partial^{-2} \bar{u} \partial^{-2}\right)
\end{aligned}
$$

with $D_{x}:=\partial$ and $\bar{u}=\left(u_{x x}+1 / 3\right)$, with respect to which the Ostrovsky-Vakhnenko hydrodynamic equation (87) is equivalent to a suitable bi-Hamiltonian flow on the functional manifold $M$. Also analyzed by means of the devised differential-algebraic tools is the Lax integrability of the interesting generalized Ostrovsky-Vakhnenko type system of evolution equations

$$
\begin{aligned}
& \left(D_{t} u+\partial^{-1} u\right)=z, \\
& D_{t} z=-u^{2} / 2
\end{aligned}
$$

on a $2 \pi$-periodic functional manifold

$$
M_{2} \subset C^{\infty}(\mathbb{R} / 2 \pi \mathbb{Z} ; \mathbb{R})^{2} .
$$

\subsection{Integrability of a Generalized Riemann Hydrodynamic System}

\subsubsection{New Generalization of the Riemann Hydrodynamic Hierarchy}

In this section we shall study the complete integrability of the dispersiveness Riemann type hydrodynamic flow (80)

$$
D_{t}^{N-1} u=\bar{z}_{x}^{2}, \quad D_{t} \bar{z}=0
$$

on a $2 \pi$-periodic functional manifold

$$
\bar{M}_{N} \subset C^{\infty}\left(\mathbb{R} / 2 \pi \mathbb{Z} ; \mathbb{R}^{N}\right),
$$

where $N \in \mathbb{N}$ is an arbitrary natural number, the vector $\left(u, D_{t} u, D_{t}^{2} u, \cdots, D_{t}^{N-1} u, \bar{z}\right)^{\top} \in \bar{M}_{N}$, the differentiations $D_{x}:=\partial / \partial x, \quad D_{t}:=\partial / \partial t+u \partial / \partial x$ satisfy, as above, the Lie-algebraic commutator relationship (77) and $t \in \mathbb{R}$ is an evolution parameter. The system can be considered as a slight generalization of the dispersiveness Riemann hydrodynamic system suggested recently by M. Pavlov and D. Holm in the form

$$
D_{t}^{N-1} u=\bar{z}, \quad D_{t} \bar{z}=0
$$

for $N \in \mathbb{N}$ and extensively studied in [8,9,32,35,37,41], where it was proved that it is a Lax integrable bi-Hamiltonian flow on the manifold $\bar{M}_{N}$ and possesses an infinite hierarchy of mutually commuting dispersive Lax integrable Hamiltonian flows.

For $N=2$ it is well known $[10,12]$ that the system (92) is a smooth Lax integrable bi-Hamiltonian flow on the $2 \pi$-periodic functional manifold $\bar{M}_{2}$, whose Lax representation is given by the compatible linear system

$$
\begin{aligned}
& D_{x} f=\left(\begin{array}{cc}
\bar{z}_{x} & 0 \\
-\lambda\left(u+u_{x} / \bar{z}_{x}\right) & -\bar{z}_{x x} / \bar{z}_{x}
\end{array}\right) f, \\
& D_{t} f=\left(\begin{array}{cc}
0 & 0 \\
-\lambda \bar{z}_{x} & u_{x}
\end{array}\right) f,
\end{aligned}
$$

where $f \in C^{\infty}\left(\mathbb{R}^{2} ; \mathbb{R}^{2}\right)$ and $\lambda \in \mathbb{R}$ is an arbitrary spectral parameter.

Our focus here is an investigation of the Lax integrability of the Riemann type hydrodynamic system (92) for $N=3$ on a $2 \pi$-periodic functional manifold $\bar{M}_{3} \subset C^{\infty}\left(\mathbb{R} / 2 \pi \mathbb{Z} ; \mathbb{R}^{3}\right)$ for a vector $(u, v, \bar{z})^{\top} \in \bar{M}_{3}$. We treat this problem in the following extended form:

$$
D_{t} u=v, D_{t} v=\bar{z}_{x}^{2}, D_{t} \bar{z}=0 .
$$

The flow (95) can be recast as a one on a $2 \pi$-periodic functional manifold $M_{3} \subset C^{\infty}\left(\mathbb{R} / 2 \pi \mathbb{Z} ; \mathbb{R}^{3}\right)$ for a vector $(u, v, z)^{\top} \in M_{3}$ as

$$
D_{t} u=v, D_{t} v=z, D_{t} z=-2 z u_{x},
$$

where, for further convenience, we have made the change of variables: $z:=\bar{z}_{x}^{2}$. We will also use the form of the flow (96):

$$
\left(\begin{array}{c}
\mathrm{d} u / \mathrm{d} t \\
\mathrm{~d} v / \mathrm{d} t \\
\mathrm{~d} z / \mathrm{d} t
\end{array}\right)=K[u, v, z]:=\left(\begin{array}{c}
v-u u_{x} \\
z-u v_{x} \\
-2 u_{x} z-u z_{x}
\end{array}\right)
$$

defining a standard smooth dynamical system on the infinite-dimensional functional manifold $M_{3}$, where $K: M_{3} \rightarrow T\left(M_{3}\right)$ is the corresponding vector field on $M_{3}$.

In the sequel, we shall prove by means of gradientholonomic and differential algebraic tools Proposition 4, stating the Lax integrability of the dynamical system (97), in particular, we will devise an effective approach for constructing its exact Lax representation and related compatible Poissonian structures.

\subsubsection{Symplectic Gradient-Holonomic Integrability Analysis: Poissonian Structure on $\boldsymbol{M}_{3}$}

Our first steps in proving Proposition 4 are fashioned using the symplectic gradient-holonomic method, which 
takes us a long way towards the desired result.

By employing the symplectic gradient-holonomic approach $[10,12,48]$ to study the integrability of smooth nonlinear dynamical systems on functional manifolds, one can find a set of conservation laws for (97) by constructing analytical solutions $\varphi:=\varphi[u, v, z] \in T^{*}\left(M_{3}\right)$ to the functional Lax gradient Equation:

$$
\mathrm{d} \varphi / \mathrm{d} t+K^{\prime, *} \varphi=\operatorname{grad} \mathcal{L},
$$

where $\varphi^{\prime}=\varphi^{\prime, *}, \quad \mathcal{L} \in D\left(M_{3}\right)$ is a suitable Lagrangian functional and the linear operator

$$
K^{\prime, *}: T^{*}\left(M_{3}\right) \rightarrow T^{*}\left(M_{3}\right)
$$

is the adjoint with respect to the standard convolution $(\cdot, \cdot)$ on $T^{*}\left(M_{3}\right) \times T\left(M_{3}\right)$, of the Fréchet derivative of a nonlinear mapping $K: M_{3} \rightarrow T\left(M_{3}\right)$; namely,

$$
K^{\prime, *}=\left(\begin{array}{ccc}
u D_{x} & -v_{x} & z_{x}+2 z D_{x} \\
1 & u_{x}+u D_{x} & 0 \\
0 & 1 & -u_{x}+u D_{x}
\end{array}\right) .
$$

The Lax gradient Equation (98) can be, owing to (80), rewritten as

$$
D_{t} \varphi+k[u, v, z] \varphi=\operatorname{grad} \mathcal{L},
$$

where the matrix operator is

$$
k[u, v, z]:=\left(\begin{array}{ccc}
0 & -v_{x} & z_{x}+2 z D_{x} \\
1 & u_{x} & 0 \\
0 & 1 & -u_{x}
\end{array}\right) .
$$

The first vector elements

$$
\begin{aligned}
& \varphi_{\vartheta}[u, v, z]=\left(z-u v_{x},-v+u u_{x}, u\right), \mathcal{L}_{\vartheta}=0 \\
& \varphi_{\eta}[u, v, z]=\left(v_{x},-u_{x},-1\right)^{\top}, \mathcal{L}_{\eta}=0, \\
& \varphi_{0}[u, v, z] \\
& =\left(-\left(u_{x} z^{-1 / 2}\right)_{x},\left(z^{-1 / 2}\right)_{x},\left(v_{x} / 2-u_{x}^{2} / 4\right) z^{-3 / 2}\right)^{\top}, \\
& \mathcal{L}_{0}=0,
\end{aligned}
$$

as can be easily checked, are solutions of the functional Equation (100). From an application of the standard Volterra homotopy formula

$$
H:=\int_{0}^{1} \mathrm{~d} \mu\left(\varphi[\mu u, \mu v, \mu z],(u, v, z)^{\top}\right),
$$

one finds the conservation laws for (80); namely,

$$
\begin{aligned}
& H_{\eta}=\frac{1}{2} \int_{0}^{2 \pi} \mathrm{d} x\left(2 u z-v^{2}-u^{2} v_{x}\right), \\
& H_{\vartheta}:=\int_{0}^{2 \pi} \mathrm{d} x\left(u v_{x} / 2-v u_{x} / 2-z\right), \\
& H_{0}:=\frac{1}{2} \int_{0}^{2 \pi} \mathrm{d} x\left(u_{x}^{2}-2 v_{x}\right) z^{-1 / 2} .
\end{aligned}
$$

It is now quite easy, making use of the conservation laws (104), to construct a Poissonian structure $\vartheta: T^{*}\left(M_{3}\right) \rightarrow T\left(M_{3}\right)$ for the dynamical system (97). If we use the representations

$$
\begin{aligned}
H_{\vartheta} & =\int_{0}^{2 \pi} \mathrm{d} x\left(u v_{x} / 2-v u_{x} / 2-z\right) \\
& :=\left(\psi_{\vartheta},\left(u_{x}, v_{x}, z_{x}\right)^{\top}\right), \\
\psi_{\vartheta} & :=\left(-v / 2, u / 2, z^{-1 / 2} D_{x}^{-1} z^{1 / 2} / 2\right)^{\top},
\end{aligned}
$$

it follows that the vector $\psi_{\vartheta} \in T^{*}\left(M_{3}\right)$ satisfies the Lax gradient Equation (100):

$$
D_{t} \psi_{\vartheta}+k[u, v, z] \psi_{\vartheta}=\operatorname{grad} \mathcal{L}_{\vartheta},
$$

where the Lagrangian function $\mathcal{L}_{\vartheta}=\left(\psi_{\vartheta}, K\right)-H_{\vartheta}$. Thus, based on the inverse co-symplectic functional expression

$$
\vartheta^{-1}:=\psi_{\vartheta}^{\prime}-\psi_{\vartheta}^{\prime * *}=\left(\begin{array}{ccc}
0 & -1 & 0 \\
1 & 0 & 0 \\
0 & 0 & z^{-1 / 2} D_{x}^{-1} z^{-1 / 2} / 2
\end{array}\right)
$$

one readily obtains the linear co-symplectic operator on the manifold $M_{3}$ :

$$
\vartheta:=\left(\begin{array}{ccc}
0 & 1 & 0 \\
-1 & 0 & 0 \\
0 & 0 & 2 z^{1 / 2} D_{x} z^{1 / 2}
\end{array}\right),
$$

which is the corresponding Poissonian operator for the dynamical system (80). It is also important to observe that the dynamical system (80) is a Hamiltonian flow on the functional manifold $M_{3}$ with respect to the Poissonian structure (108).

$$
K[u, v, z]=-\vartheta_{\operatorname{grad}} H_{\eta} .
$$

\subsubsection{Poissonian Structure on $\bar{M}_{3}$}

In what follows, we shall find it convenient to construct other Poissonian structures for dynamical system (95) on the manifold $\bar{M}_{3}$, rewritten in the equivalent form

$$
\frac{\mathrm{d}}{\mathrm{d} t}\left(\begin{array}{c}
u \\
v \\
\bar{z}
\end{array}\right)=\bar{K}[u, v, \bar{z}]:=\left(\begin{array}{c}
v-u u_{x} \\
\bar{z}_{x}^{2}-u v_{x} \\
0
\end{array}\right),
$$

where $\bar{K}: \bar{M}_{3} \rightarrow T\left(\bar{M}_{3}\right)$ is the corresponding vector field on $\bar{M}_{3}$. To proceed, we need to obtain additional solutions to the Lax gradient Equation (100) on the functional manifold $\bar{M}_{3}$

$$
D_{t} \bar{\psi}+\bar{k}[u, v, z] \bar{\psi}=\operatorname{grad} \overline{\mathcal{L}},
$$

where the matrix operator is

$$
\bar{k}[u, v, \bar{z}]:=\left(\begin{array}{ccc}
0 & -v_{x} & -\bar{z}_{x} \\
1 & u_{x} & 0 \\
0 & -2 \partial \bar{z}_{x} & u_{x}
\end{array}\right),
$$


and which we may rewrite in the component form

$$
\begin{aligned}
& D_{t} \bar{\psi}^{(1)}=v_{x} \bar{\psi}^{(2)}+\bar{z}_{x} \bar{\psi}^{(3)}+\delta \overline{\mathcal{L}} / \delta u, \\
& D_{t} \bar{\psi}^{(2)}=-\bar{\psi}^{(1)}-u_{x} \bar{\psi}^{(2)}+\delta \overline{\mathcal{L}} / \delta v, \\
& D_{t} \bar{\psi}^{(3)}=2\left(\bar{z}_{x} \bar{\psi}^{(2)}\right)_{x}-u_{x} \bar{\psi}^{(3)}+\delta \overline{\mathcal{L}} / \delta \bar{z},
\end{aligned}
$$

where the vector

$$
\bar{\psi}:=\left(\bar{\psi}^{(1)}, \bar{\psi}^{(2)}, \bar{\psi}^{(3)}\right)^{\top} \in T^{*}\left(\bar{M}_{3}\right) .
$$

As a simple consequence of (113), one obtains the following system of differential relationships:

$$
\begin{aligned}
D_{t}^{3} \tilde{\psi}^{(2)}= & -2 \bar{z}_{x}^{2} \tilde{\psi}_{x}^{(2)}+D_{t}^{2} \partial^{-1}(\delta \overline{\mathcal{L}} / \delta v) \\
& -\partial^{-1}\left\langle\operatorname{grad} \overline{\mathcal{L}},\left(u_{x}, v_{x}, \bar{z}_{x}\right)^{\top}\right\rangle, \\
D_{t} \tilde{\psi}^{(2)}= & -\tilde{\psi}^{(1)}+\partial^{-1}(\delta \overline{\mathcal{L}} / \delta v), \\
D_{t} \tilde{\psi}^{(3)}= & 2 \bar{z}_{x} \tilde{\psi}_{x}^{(2)}+\partial^{-1}(\delta \overline{\mathcal{L}} / \delta \bar{z}) .
\end{aligned}
$$

Here we have defined

$$
\left(\bar{\psi}^{(1)}, \bar{\psi}^{(2)}, \bar{\psi}^{(3)}\right)^{\top}:=\left(\tilde{\psi}_{x}^{(1)}, \tilde{\psi}_{x}^{(2)}, \tilde{\psi}_{x}^{(3)}\right)^{\top}
$$

and made use of the commutator relationship for differentiations $D_{t}$ and $D_{x}$ :

$$
\left[D_{t}, \alpha^{-1} D_{x}\right]=0
$$

which holds for the function $\alpha:=1 / \bar{Z}_{x}$, where $D_{t} \bar{z}=0$. It therefore follows that after solving the first equation of system (114), and one can recursively solve the remaining two equations. In particular, it is easy to see that the three vector elements

$$
\begin{aligned}
& \tilde{\psi}_{0}=\left(-v, u,-2 \bar{z}_{x}\right)^{\top}, \overline{\mathcal{L}}_{0}=0 ; \\
& \tilde{\psi}_{\theta}=\left(-u_{x} / \bar{z}_{x}, 1 / \bar{z}_{x},\left(u_{x}^{2}-2 v_{x}\right) /\left(2 \bar{z}_{x}^{2}\right)\right)^{\top}, \\
& \overline{\mathcal{L}}_{\theta}=0 ; \\
& \tilde{\psi}_{\eta}=\left(u / 2,-x / 2, \partial^{-1}\left[\left(2 v_{x}-u_{x}^{2}\right) /\left(2 \bar{z}_{x}\right)\right]\right)^{\top}, \\
& \overline{\mathcal{L}}_{\eta}=\left(D_{x} \tilde{\psi}_{\eta}, \bar{K}\right)-H_{\vartheta},
\end{aligned}
$$

are solutions of the system (114). The first two elements of (116) lead to the Volterra symmetric vectors

$$
\begin{aligned}
\bar{\psi}_{0} & =D_{x} \tilde{\psi}_{0}, \bar{\psi}_{\theta}=D_{x} \tilde{\psi}_{\theta} \\
& \in T^{*}\left(\bar{M}_{3}\right): \bar{\psi}_{0}^{\prime}=\bar{\psi}_{0}^{\prime, *}, \bar{\psi}_{\theta}^{\prime}=\bar{\psi}_{\theta}^{\prime, *}
\end{aligned}
$$

entailing the trivial conservation laws

$$
\left(\bar{\psi}_{0}, \bar{K}\right)=0=\left(\bar{\psi}_{\theta}, \bar{K}\right) \text {. }
$$

The third element of (116) gives rise to the Volterra asymmetric vector $\bar{\psi}_{\eta}:=D_{x} \tilde{\psi}_{\eta}: \bar{\psi}_{\eta}^{\prime} \neq \bar{\psi}_{\eta}^{\prime, *}$, entailing the following inverse co-symplectic functional expression:

$$
\begin{aligned}
\bar{\eta}^{-1}:=\bar{\psi}_{\eta}^{\prime}-\bar{\psi}_{\eta}^{\prime, *} & -\partial \frac{u_{x}}{\bar{z}_{x}} \\
& =\left(\begin{array}{ccc}
0 & 0 & \partial \frac{1}{\bar{z}_{x}} \\
0 & 0 & u_{x} \\
-\frac{u_{x}}{\bar{z}_{x}} \partial & \frac{1}{\bar{z}_{x}} \partial & \frac{u_{x}}{2 \bar{z}_{x}}-\frac{v_{x}}{\bar{z}_{x}} \partial \frac{1}{\bar{Z}_{x}}-\frac{1}{\bar{z}_{x}} \partial \frac{v_{x}}{\bar{z}_{x}}
\end{array}\right) .
\end{aligned}
$$

Correspondingly, the Poissonian operator

$$
\bar{\eta}: T^{*}\left(\bar{M}_{3}\right) \rightarrow T\left(\bar{M}_{3}\right)
$$

is

$$
\bar{\eta}=\left(\begin{array}{ccc}
\partial^{-1} & u_{x} \partial^{-1} & 0 \\
\partial^{-1} u_{x} & v_{x} \partial^{-1}+\partial^{-1} v_{x} & \partial^{-1} \bar{z}_{x} \\
0 & \bar{z}_{x} \partial^{-1} & 0
\end{array}\right),
$$

subject to which the following Hamiltonian representation

$$
\bar{K}[u, v, \bar{z}]=-\left.\bar{\eta} \operatorname{grad} H_{\eta}\right|_{z=z_{x}^{2}}
$$

holds on the manifold $\bar{M}_{3}$.

\subsubsection{Hamiltonian Integrability Analysis}

Next, we return to our integrability analysis of the dynamical system (97) on the functional manifold $M_{3}$. It is easy to recalculate the form of the Poissonian operator (118) on the manifold $\bar{M}_{3}$ to that acting on the manifold $M_{3}$, giving rise to the second Hamiltonian representation of (97):

$$
K[u, v, z]=-\eta \operatorname{grad} H_{\vartheta},
$$

where $\eta: T^{*}\left(M_{3}\right) \rightarrow T\left(M_{3}\right)$ is the corresponding Poissonian operator. As a next important point, the Poissonian operators (108) and (118) are compatible $[1,3,10,12]$ on the manifold $\bar{M}_{3}$; that is, the operator pencil $(\vartheta+\lambda \eta): T^{*}\left(M_{3}\right) \rightarrow T\left(M_{3}\right)$ is also Poissonian for arbitrary $\lambda \in \mathbb{R}$. As a consequence, any operator of the form

$$
\vartheta_{n}:=\vartheta\left(\vartheta^{-1} \eta\right)^{n}
$$

for all $n \in \mathbb{Z}$ is Poissonian on the manifold $M_{3}$. Using now the homotopy formula (103) and recursion property of the Poissonian pair (109) and (118), it is easy to construct the related infinite hierarchy of mutually commuting conservation laws

$$
\begin{aligned}
& \gamma_{j}=\int_{0}^{1} \mathrm{~d} s\left(\operatorname{grad} \gamma_{j}[s u, s v, s z],(u, v, z)^{\top}\right), \\
& \operatorname{grad} \gamma_{j}[u, v, z]:=\Lambda^{j} \operatorname{grad} H_{\eta},
\end{aligned}
$$

for the dynamical system (97), where $j \in \mathbb{Z}_{+}$and

$$
\Lambda:=\vartheta^{-1} \eta: T^{*}\left(M_{3}\right) \rightarrow T^{*}\left(M_{3}\right)
$$


is the corresponding recursion operator, which satisfies the so called associated Lax commutator relationship

$$
\mathrm{d} \Lambda / \mathrm{d} t=\left[\Lambda, K^{\prime, *}\right] .
$$

In the course of the above analysis and observations, we have proved the following result.

Proposition 6 The Riemann hydrodynamic system (97) is a bi-Hamiltonian dynamical system on the functional manifold $M_{3}$ with respect to the compatible Poissonian structures $\vartheta, \eta: T^{*}\left(M_{3}\right) \rightarrow T\left(M_{3}\right)$

$$
\begin{aligned}
\vartheta & :=\left(\begin{array}{ccc}
0 & 1 & 0 \\
-1 & 0 & 0 \\
0 & 0 & 2 z^{1 / 2} D_{x} z^{1 / 2}
\end{array}\right), \\
\eta & :=\left(\begin{array}{ccc}
\partial^{-1} & u_{x} \partial^{-1} & 0 \\
\partial^{-1} u_{x} & v_{x} \partial^{-1}+\partial^{-1} v_{x} & \partial^{-1} z_{x}-2 z \\
0 & z_{x} \partial^{-1}+2 z & 0
\end{array}\right)
\end{aligned}
$$

and possesses an infinite hierarchy of mutually commuting conservation laws (122).

Concerning the existence of an additional infinite and parametrically $\mathbb{R} \ni \lambda$-ordered hierarchy of conservation laws for the dynamical system (92), it is instructive to consider the dispersive nonlinear dynamical system

$$
\begin{aligned}
& (\mathrm{d} u / \mathrm{d} \tau, \mathrm{d} v / \mathrm{d} \tau, \mathrm{d} z / \mathrm{d} \tau)^{\top}=\tilde{K}[u, v, z] \\
& :=-\vartheta \operatorname{grad} H_{0}[u, v, z] \\
& =\left(-\left(z^{-1 / 2}\right)_{x},-\left(u_{x} z^{-1 / 2}\right)_{x}, z^{1 / 2}\left(\frac{u_{x}^{2}-2 v_{x}}{2 z}\right)_{x}\right)^{\top} .
\end{aligned}
$$

By solving the corresponding Lax equation

$$
\mathrm{d} \tilde{\varphi} / \mathrm{d} t+\tilde{K}^{\prime, *} \tilde{\varphi}=0
$$

for an element $\tilde{\varphi} \in T^{*}\left(M_{3}\right)$ in a suitably chosen asymptotic form, one can construct an infinite ordered hierarchy of conservation laws for (92), which we will not delve into here. This hierarchy and the existence of an infinite and parametrically $\mathbb{R} \ni \lambda$-ordered hierarchy of conservation laws for the Riemann type dynamical system (92) provided compelling indications that it is completely integrable in the sense of Lax on the functional manifold $M_{3}$. We shall study the complete integrability in the next section using rather powerful differentialalgebraic tools that were devised recently in [8,9,37].

\subsection{D-A Integrability Analysis for $N=3$}

Consider a polynomial differential ring

$$
\mathcal{K}\{u\} \subset \mathcal{K}:=\mathbb{R}\{\{x, t\}\}
$$

generated by a fixed functional variable $u \in \mathbb{R}\{\{x, t\}\}$ and invariant with respect to two differentiations $D_{x}:=\partial / \partial x$ and $D_{t}:=\partial / \partial t+u \partial / \partial x$ that satisfy the Lie-algebraic commutator relationship (77) together with the constraint (96) expressed in the differential-algebraic functional form

$$
D_{t}^{3} u=-2 D_{t}^{2} u D_{x} u
$$

Since the Lax representation for the dynamical system (97) can be interpreted $[8,10]$ as the existence of a finite-dimensional invariant ideal $\mathcal{I}\{u\} \subset \mathcal{K}\{u\}$ realizing the corresponding finite-dimensional representation of the Lie-algebraic commutator relationship (127), this ideal can be constructed as

$$
\begin{gathered}
\mathcal{I}\{u\}:=\left\{\lambda^{2} u f_{1}+\lambda v f_{2}+z^{1 / 2} f_{3} \in \mathcal{K}\{u\}: f_{j} \in \mathcal{K},\right. \\
1 \leq j \leq 3, \lambda \in \mathbb{R}\},
\end{gathered}
$$

where $v=D_{t} u, z=D_{t}^{2} u$ and $\lambda \in \mathbb{R}$ is an arbitrary real parameter. To find finite-dimensional representations of the $D_{x}$ - and $D_{t}$-differentiations, it is necessary [8] first to find the $D_{t}$-invariant kernel $\operatorname{ker} D_{t} \subset \mathcal{I}\{u\}$ and next to check its invariance with respect to the $D_{x}$-differentiation. It is easy to show that

$$
\operatorname{ker} D_{t}=\left\{f \in \mathcal{K}^{3}: D_{t} f=q(\lambda) f, \lambda \in \mathbb{R}\right\},
$$

where the matrix $q(\lambda):=q[u, v, z ; \lambda] \in \operatorname{End} \mathcal{K}\{u\}^{3}$ is given as

$$
q(\lambda)=\left(\begin{array}{ccc}
0 & 0 & 0 \\
-\lambda & 0 & 0 \\
0 & -\lambda z_{x} & u_{x}
\end{array}\right)
$$

To obtain the corresponding representation of the $D_{x}$-differentiation in the space $\mathcal{K}^{3}$, it suffices to find a matrix $l(\lambda):=l[u, v, z ; \lambda] \in \operatorname{End} \mathcal{K}\{u\}^{3}$ that

$$
D_{x} f=l(\lambda) f
$$

for $f \in \mathcal{K}\{u\}^{3}$ and the related ideal

$$
\begin{aligned}
& \mathcal{R}\{u\}:= \\
& \left\{\langle g, f\rangle_{\mathcal{K}^{3}}: f \in \operatorname{ker} D_{t} \subset \mathcal{K}^{3}\{u\}, g \in \mathcal{K}\{u\}^{3}\right\}
\end{aligned}
$$

is $D_{x}$-invariant with respect to the differentiation (131). Straightforward calculations using this invariance condition then yield the following matrix

$$
l(\lambda)=\left(\begin{array}{ccc}
\lambda^{2} u \sqrt{z} & \lambda v \sqrt{z} & z \\
-\lambda^{3} t u \sqrt{z} & -\lambda^{2} t v \sqrt{z} & -\lambda t z \\
\lambda^{4}\left(t u v-u^{2}\right)-\lambda^{2} u_{x} / \sqrt{z} & -\lambda v_{x} / \sqrt{z}+\lambda^{3}\left(t v^{2}-u v\right) & \lambda^{2} \sqrt{z}(u-t v)-z_{x} / 2 z
\end{array}\right) .
$$


Remark 1 Simple analogs of the above differentialalgebraic calculations for the case $N=2$ lead readily to the corresponding Riemann type hydrodynamic system

$$
D_{t} u=\bar{z}_{x}^{2}, \quad D_{t} \bar{z}=0
$$

on the functional manifold $\bar{M}_{2}$, which possesses the following matrix Lax representation:

$$
\begin{aligned}
& D_{t} f=\left(\begin{array}{cc}
0 & 0 \\
-\lambda \bar{z}_{x} & u_{x}
\end{array}\right) f, \\
& D_{x} f=\left(\begin{array}{cc}
\bar{z}_{x} & 0 \\
-\lambda\left(u+u_{x} / \bar{z}_{x}\right) & -\bar{z}_{x x} / \bar{z}_{x}
\end{array}\right) f,
\end{aligned}
$$

where $\lambda \in \mathbb{R}$ is an arbitrary spectral parameter and $f \in C^{\infty}\left(\mathbb{R}^{2} ; \mathbb{R}^{2}\right)$.

As one can readily see, these differential-algebraic results provide a direct proof of Proposition 4 describing the integrability of system (97) for $N=3$. The matrices (133) are not of standard form since they depend explicitly on the temporal evolution parameter $t \in \mathbb{R}$. Nonetheless, the matrices (130) and (133) satisfy for all $\lambda \in \mathbb{R}$ the well-known Zakharov-Shabat type compatibility condition

$$
D_{t} l(\lambda)=[q(\lambda), l(\lambda)]+D_{x} q(\lambda)-u_{x} l(\lambda),
$$

which follows from the Lax type relationships (129) and (131)

$$
D_{t} f=q(\lambda) f, \quad D_{x} f=l(\lambda) f
$$

and the commutator condition (127). Moreover, taking into account that the dynamical system (97) has a compatible Poissonian pair (108) and (118) depending only on the variables $(u, v, z)^{\top} \in M_{3}$ and not depending on the temporal variable $t \in \mathbb{R}$, one can certainly assume that it also possesses a standard autonomous Lax representation, which can possibly be found by means of a suitable gauge transformation of (137). We plan to pursue this line of analysis in a forthcoming paper.

\subsection{Integrability of Ostrovsky-Vakhnenko Equation}

\subsubsection{An Introduction and Problem Description}

In 1998 V. O. Vakhnenko investigated high-frequency perturbations in a relaxing barotropic medium. He discovered that this phenomenon is described by a new non-linear evolution equation. Later it was proved that this equation is equivalent to the reduced Ostrovsky equation [44], which describes long internal waves in a rotating ocean. The nonlinear integro-differential Ostrovsky-Vakhnenko equation

$$
u_{t}=-u u_{x}-D_{x}^{-1} u
$$

on the real axis $\mathbb{R}$ for a smooth function $u \in C^{\infty}\left(\mathbb{R}^{2} ; \mathbb{R}\right)$, where $D_{x}^{-1}$ is the inverse-differential operator to $D_{x}:=\partial / \partial x$, can be derived [45] as a special case of the Whitham type equation

$$
u_{t}=-u u_{x}+\int_{\mathbb{R}} K(x, y) u_{y} \mathrm{~d} y .
$$

Here the generalized kernel

$$
K(x, y):=\frac{1}{2}|x-y|, x, y \in \mathbb{R}
$$

and $t \in \mathbb{R}$ is an evolution parameter. Various analytical properties of (138) and related Equations were analyzed in articles [44-46,49], the corresponding Lax integrability was proved in [43].

Recently, J. C. Brunelli and S. Sakovich [42] demonstrated that the Ostrovsky-Vakhnenko Equation is a reduction of the well known Camassa-Holm Equation making it possible to construct the corresponding compatible Poisson structures for (138), but in a complicated nonpolynomial form.

In the present work we will reanalyze the integrability of Equation (138) from the gradient-holonomic [10,12,48], symplectic and formal differential-algebraic points of view. As a result, we will re-derive the Lax representation for the Ostrovsky-Vakhnenko Equation (138) and construct the related simple compatible polynomial Poisson structures and an infinite hierarchy of conservation laws.

\subsubsection{Gradient-Holonomic Integrability Analysis}

Consider the nonlinear Ostrovsky-Vakhnenko Equation (138) as a a nonlinear dynamical system

$$
\mathrm{d} u / \mathrm{d} t=K[u]:=-u u_{x}-D_{x}^{-1} u
$$

on the smooth $2 \pi$-periodic functional manifold

$$
M:=\left\{u \in C^{\infty}(\mathbb{R} / 2 \pi \mathbb{Z} ; \mathbb{R}): \int_{0}^{2 \pi} u \mathrm{~d} x=0\right\},
$$

where $K: M \rightarrow T(M)$ is the corresponding well-defined smooth vector field on $M$.

We shall first show that the dynamical system (140) on manifold $M$ possesses an infinite hierarchy of conservation laws as a necessary condition for its integrability. For this we need to construct a solution to the Lax gradient equation

$$
\varphi_{t}+K^{\prime, *} \varphi=0,
$$

in the special asymptotic form

$$
\varphi=\exp \left[-\lambda t+D_{x}^{-1} \sigma(x ; \lambda)\right]
$$

where, by definition, a linear operator

$$
K^{\prime, *}: T^{*}(M) \rightarrow T^{*}(M)
$$

is adjoint with respect to the standard convolution $(\cdot, \cdot)$ on $T^{*}(M) \times T(M)$; the Fréchet-derivative of a nonlin- 
ear mapping $K: M \rightarrow T(M)$ :

$$
K^{\prime, *}=u D_{x}+D_{x}^{-1}
$$

and, respectively,

$$
\sigma(x ; \lambda) \simeq \sum_{j \in \mathbb{Z}_{+}} \sigma_{j}[u] \lambda^{-j},
$$

as $|\lambda| \rightarrow \infty$ with some local functionals

$$
\sigma_{j}: M \rightarrow C^{\infty}(\mathbb{R} / 2 \pi \mathbb{Z} ; \mathbb{R})
$$

on $M$ for all $j \in \mathbb{Z}_{+}$.

By substituting (143) into (142), one easily obtains the following recurrent sequence of functional relationships

$$
\begin{aligned}
& \sigma_{j, t}+\sum_{k \leq j} \sigma_{j-k}\left(u \sigma_{k}+D_{x}^{-1} \sigma_{k, t}\right)-\sigma_{j+1} \\
& +\left(u \sigma_{j}\right)_{x}+\delta_{j, 0}=0
\end{aligned}
$$

for all $j+1 \in \mathbb{Z}_{+}$modulo the Equation (140). By means of standard calculations one finds that this recurrent sequence is solvable and

$$
\begin{aligned}
& \sigma_{0}[u]=0, \sigma_{1}[u]=1, \sigma_{2}[u]=u_{x}, \\
& \sigma_{3}[u]=0, \sigma_{4}[u]=u_{t}+2 u u_{x}, \\
& \sigma_{5}[u]=3 / 2\left(u^{2}\right)_{x t}+u_{t t}+2 / 3\left(u^{3}\right)_{x x}-u_{x} D_{x}^{-1} u
\end{aligned}
$$

and so on. It is easy check that all of functionals

$$
\gamma_{j}:=\int_{0}^{2 \pi} \sigma_{j}[u] d x
$$

are conservation laws on the manifold $M$, that is $\mathrm{d} \gamma_{j} / \mathrm{d} t=0$ for $j \in \mathbb{Z}_{+}$with respect to the dynamical system (140). For instance, if $j=5$ one obtains:

$$
\begin{aligned}
\gamma_{5} & :=\int_{0}^{2 \pi} \sigma_{5}[u] \mathrm{d} x \\
& =\int_{0}^{2 \pi}\left[3 / 2\left(u^{2}\right)_{x t}+u_{t t}+2 / 3\left(u^{3}\right)_{x x}-u_{x} D_{x}^{-1} u\right] \mathrm{d} x \\
& =\frac{\mathrm{d}^{2}}{\mathrm{~d} t^{2}} \int_{0}^{2 \pi} u_{t t} \mathrm{~d} x-\int_{0}^{2 \pi} u_{x} D_{x}^{-1} u \mathrm{~d} x \\
& =\frac{\mathrm{d}^{2}}{\mathrm{~d} t^{2}} \int_{0}^{2 \pi} u \mathrm{~d} x-\left.u D_{x}^{-1} u\right|_{0} ^{2 \pi}+\int_{0}^{2 \pi} u^{2} \mathrm{~d} x=\int_{0}^{2 \pi} u^{2} \mathrm{~d} x,
\end{aligned}
$$

and

$$
\begin{aligned}
\mathrm{d} \gamma_{5} / \mathrm{d} t & =2 \int_{0}^{2 \pi} u u_{t} \mathrm{~d} x=-2 \int_{0}^{2 \pi} u\left(u u_{x}+D_{x}^{-1} u\right) \mathrm{d} x \\
& =-2 \int_{0}^{2 \pi} u D_{x}^{-1} u \mathrm{~d} x-\int_{0}^{2 \pi}\left[\left(D_{x}^{-1} u\right)^{2}\right]_{x} \mathrm{~d} x \\
& =\left.\left(D_{x}^{-1} u\right)^{2}\right|_{0} ^{2 \pi}=0,
\end{aligned}
$$

since, owing to the constraint (141), the integrals $\left.\left(D_{x}^{-1} u\right)\right|_{0} ^{2 \pi}=0$.

The result above suggests that the dynamical system (140) on the functional manifold $M$ is an integrable
Hamiltonian system.

First, we will show that this dynamical system is a Hamiltonian flow

$$
\mathrm{d} u / \mathrm{d} t=-\vartheta \operatorname{grad} H[u]
$$

with respect to some Poisson structure

$$
\vartheta: T^{*}(M) \rightarrow T(M)
$$

and a Hamiltonian function $H \in \mathcal{D}(M)$. Using on the standard symplectic techniques $[1,3,10,12]$, consider the conservation law (149) and present it in the scalar "momentum" form

$$
\begin{aligned}
-1 / 2 \gamma_{5} & =\frac{1}{2} \int_{0}^{2 \pi} u_{x} D_{x}^{-1} u \mathrm{~d} x \\
& =\left(1 / 2 D_{x}^{-1} u, u_{x}\right):=\left(\psi, u_{x}\right)
\end{aligned}
$$

with the co-vector $\psi:=1 / 2 D_{x}^{-1} u \in T^{*}(M)$ and calculate the corresponding co-Poissonian structure

$$
\vartheta^{-1}:=\psi^{\prime}-\psi^{\prime, *}=D_{x}^{-1},
$$

or the Poissonian structure

$$
\vartheta=D_{x} .
$$

This operator $\vartheta=D_{x}: T^{*}(M) \rightarrow T(M)$ is really Poissonian for (140) since the following determining symplectic condition

$$
\psi_{t}+K^{\prime, *} \psi=\operatorname{grad} \mathcal{L}
$$

holds for the Lagrangian function

$$
\mathcal{L}=\frac{1}{12} \int_{0}^{2 \pi} u^{3} \mathrm{~d} x .
$$

As a result of (155), one readily infers that

$$
\mathrm{d} u / \mathrm{d} t=-\vartheta \operatorname{grad} H[u],
$$

where the Hamiltonian function

$$
\begin{aligned}
H & =(\psi, K)-\mathcal{L} \\
& =\frac{1}{2} \int_{0}^{2 \pi}\left[u^{3} / 3-\left(D_{x}^{-1} u\right)^{2} / 2\right] \mathrm{d} x
\end{aligned}
$$

is an additional conservation law of the dynamical system (140). Thus, one can formulate the following proposition.

Proposition 7 The Ostrovsky-Vakhnenko dynamical system (140) possesses an infinite hierarchy of nonlocal, in general, conservation laws (148) and is a Hamiltonian flow (157) on the manifold $M$ with respect to the Poissonian structure (154).

Remark 2 It is useful to remark here that the existence of an infinite ordered (by $\lambda$-powers) hierarchy of conservations laws (148) is a typical property $[1,3,10,12]$ of Lax integrable Hamiltonian systems that are simultaneously bi-Hamiltonian flows with respect to a corre- 
sponding pair of compatible Poissonian structures (cf. [50]).

As is well known $[1,3,10,12]$, the second Poissonian structure $\eta: T^{*}(M) \rightarrow T(M)$ on the manifold $M$ for (140), if it exists, can be calculated as

$$
\eta^{-1}:=\tilde{\psi}^{\prime}-\tilde{\psi}^{\prime, *},
$$

where a covector $\tilde{\psi} \in T^{*}(M)$ is a second solution to the determining Equation (155):

$$
\tilde{\psi}_{t}+K^{\prime, *} \tilde{\psi}=\operatorname{grad} \tilde{\mathcal{L}}
$$

for some Lagrangian functional $\tilde{\mathcal{L}} \in \mathcal{D}(M)$. It can be easily shown by means of simple but cumbersome analytical calculations based, for example, on the asymptotic small parameter method $[10,12,48]$ and on which we will not dwell upon here.

Instead of this, we shall apply the direct differential-algebraic approach to dynamical system (140) and reveal its Lax representation both in the differential scalar and canonical matrix Zakharov-Shabat forms. Moreover, we will construct the naturally related compatible polynomial Poissonian structures for the OstrovskyVakhnenko dynamical system (140) and generate an infinite hierarchy of mutually commuting nonlocal conservation laws.

\subsection{Lax Representation and Poisson Structures: A D-A Approach}

We will start by constructing of the polynomial differential ring $\mathcal{K}\{u\} \subset \mathcal{K}:=\mathbb{R}\{\{x, t\}\}$ generated by a fixed functional variable $u \in \mathbb{R}\{\{x, t\}\}$ and invariant with respect to two differentiations

$$
D_{x}:=\partial / \partial x \text { and } D_{t}:=\partial / \partial t+u \partial / \partial x,
$$

satisfying the Lie-algebraic commutator relationship (77). Since the Lax representation for the dynamical system (140) can be interpreted $[8,10]$ as the existence of a finitedimensional invariant differential ideal $\mathcal{I}_{N}\{u\} \subset \mathcal{K}\{u\}$, realizing the corresponding finite-dimensional representation of the Lie-algebraic commutator relationship (77), this ideal can be presented as

$$
\begin{aligned}
& \mathcal{I}_{N}\{u\}:= \\
& \left\{\sum_{j=0, N} g_{j} D_{x}^{j} f[u] \in \mathcal{K}\{u\}: g_{j} \in \mathcal{K}, j=\overline{0, N}\right\},
\end{aligned}
$$

where an element $f[u] \in \mathcal{K}\{u\}$ and $N \in \mathbb{Z}_{+}$are fixed. The $D_{x}$-invariance of ideal (161) will be a priori evident, if the function $f[u] \in \mathcal{K}\{u\}$ satisfies the linear differential relationship

$$
D_{x}^{N+1} f=\sum_{k=0}^{N} a_{j}[u] D_{x}^{j} f
$$

for some coefficients $a_{j}[u] \in \mathcal{K}\{u\}, \quad j=\overline{0, N}$, but its
$D_{t}$-invariance strongly depends on the element $f[u] \in \mathcal{K}\{u\}$, which can be found from the functional relationship (142) on the element

$$
\varphi[u ; \lambda]:=\operatorname{grad} \gamma(\lambda) \in \mathcal{K}\{u\}, \gamma(\lambda):=\int_{0}^{2 \pi} \sigma(x ; \lambda) \mathrm{d} x,
$$

rewritten in the following form:

$$
D_{x} D_{t} \varphi=-\varphi \text {. }
$$

From the right-hand side it follows that there exists an element $\eta:=\eta[u]=-D_{t} \varphi[u] \in \mathcal{K}\{u\}$, such that

$$
\varphi=D_{x} \eta \text {. }
$$

Upon substituting (164) into the left hand side of (163) one finds that

$$
\begin{aligned}
D_{x} D_{t} \eta-u_{x} \eta_{x} & =D_{x} D_{t} \eta-u_{x} \varphi \\
& =D_{x} D_{t} \eta-u_{x} \operatorname{grad} \gamma(\lambda) \\
& =D_{x}\left(D_{t} \eta-\gamma[u, \lambda]\right)=-\eta,
\end{aligned}
$$

where $\gamma(\lambda):=\int_{0}^{2 \pi} \gamma[u ; \lambda] \mathrm{d} x$ for a suitably chosen density element $\gamma[u ; \lambda] \in \mathcal{K}\{u\}$. As an evident result of (165) one concludes that there exists an element $\rho:=\rho[u] \in \mathcal{K}\{u\}$, such that

$$
\eta=D_{x} \rho .
$$

Turning back to the relationships (164) and (166), one concludes that the differential representation

$$
\varphi=D_{x}^{2} \rho
$$

holds.

As a further step, we can try to realize the differential ideal (161) by means of the generating element $f[u] \Rightarrow \rho[u] \in \mathcal{K}\{u\}$, defined by the relationship (167). But, as it is easy to check, this differential ideal is not finite-dimensional. So, for future calculating convenience, we will represent the element $\rho[u] \in \mathcal{K}\{u\}$ in the following natural factorized form:

$$
\rho:=\overline{f f},
$$

where elements $f, \bar{f} \in \mathcal{K}\{u\}$ satisfy the adjoint pairs of the following differential relationships:

$$
\begin{aligned}
& D_{x}^{N+1} f=\sum_{k=0}^{N} a_{j}[u] D_{x}^{j} f, \\
& (-1)^{N+1} D_{x}^{N+1} \bar{f}=\sum_{k=0}^{N}\left(-D_{x}\right)^{j}\left(a_{j}[u] \bar{f}\right),
\end{aligned}
$$

and

$$
\begin{aligned}
& D_{t} f=\sum_{j=0}^{N} b_{j}[u] D_{x}^{j} f, \\
& D_{t} \bar{f}=-u_{x} \bar{f}-\sum_{j=0}^{N}\left(-D_{x}\right)^{j}\left(b_{j}[u] \bar{f}\right),
\end{aligned}
$$

for some elements $b_{j}[u] \in \mathcal{K}\{u\}, j=\overline{0, N}$, and check 
the finite-dimensional $D_{x}$ - and $D_{t}$-invariance of the corresponding ideal (161) generated by the element $f \in \mathcal{K}\{u\}$.

Now it is easy to check by means of straightforward calculations, based on the relationship (163) and (167), that the following differential equalities

$$
\begin{aligned}
D_{x}\left(D_{t} \varphi\right)= & -\varphi, D_{x}\left(D_{t}^{2} \varphi\right)=-u_{x} \varphi-D_{t} \varphi, \\
D_{x}\left(D_{t}^{3} \varphi\right)= & u \varphi-2 u_{x} D_{t} \varphi-D_{t}^{2} \varphi, \\
D_{x}\left(D_{t}^{4} \varphi\right)= & -\left(u u_{x}+D_{x}^{-1} u\right) \varphi+\left(4 u_{x}^{2}+3 u\right) D_{t} \varphi \\
& -2 u_{x} D_{t}^{2} \varphi-D_{t}^{3} \varphi,
\end{aligned}
$$

and their consequences

$$
\begin{aligned}
& D_{t} D_{x}^{2} \rho=-\rho_{x}, \\
& D_{x}\left(D_{t} \rho_{x}\right)=u_{x} \rho_{x x}-D_{x} \rho, \\
& D_{x}^{2}\left(D_{t} \rho\right)=D_{x}\left(u_{x} D_{x} \rho-\rho\right)+u_{x x} D_{x}^{2} \rho, \cdots,
\end{aligned}
$$

hold. Taking into account the independence of the sets of functional elements

$$
\left\{f, D_{x} f, D_{x} f, \cdots, D_{x}^{N} f\right\} \subset \mathcal{K}\{u\}
$$

and

$$
\left\{\bar{f}, D_{x} \bar{f}, D_{x} \bar{f}, \cdots, D_{x}^{N} \bar{f}\right\} \subset \mathcal{K}\{u\},
$$

the relationships (172) together with (168), (169) and (170) make it possible to state the following lemma.

Lemma 2 The set (161) represents $a D_{x}$ - and $D_{t}$-invariant differential ideal in the ring $\mathcal{K}$ for all $N \geq 2$.

Proof 1 This result easily follows from the fact that for $N \geq 2$ all of the relationships (172) are compatible upon taking into account the differential expressions (168) and (170). However, for $N=1$ they are not compatible.

As a corollary of Lemma 2, in light of (161) and (170), for $N=2$, one readily finds by means of elementary calculations that the related differential ideal $\mathcal{I}_{2}\{u\}$ is to be invariant if the following differential Lax relationships hold:

$$
D_{x}^{3} f=-\mu \bar{u} f, D_{x}^{3} \bar{f}=\mu \bar{u} \bar{f},
$$

and

$$
\begin{aligned}
& D_{t} f=\mu^{-1} D_{x}^{2} f+u_{x} f, \\
& D_{t} \bar{f}=-\mu^{-1} D_{x}^{2} \bar{f}-2 u_{x} \bar{f},
\end{aligned}
$$

where $\bar{u}:=u_{x x}+1 / 3, \mu \in \mathbb{C} \backslash\{0\}$ is an arbitrary complex parameter. Moreover, they exactly coincide with those found before in [43]. The above differential relationships (173) and (174) can be equivalently rewritten in the following matrix Zakharov-Shabat type form:

$$
D_{t} h=\hat{q}[u ; \mu] h, D_{x} h=\hat{l}[u ; \mu] h,
$$

where the matrices

$$
\begin{aligned}
& \hat{q}[u ; \mu]:=\left(\begin{array}{ccc}
u_{x} & 0 & 1 / \mu \\
-1 / 3 & 0 & 0 \\
0 & -1 / 3 & -u_{x}
\end{array}\right), \\
& \hat{l}[u ; \mu]:=\left(\begin{array}{ccc}
0 & 1 & 0 \\
0 & 0 & 1 \\
-\mu \bar{u} & 0 & 0
\end{array}\right)
\end{aligned}
$$

and $h:=\left(f, D_{x} f, D_{x}^{2} f\right)^{\top} \in \mathcal{K}\{u\}^{3}$.

Furthermore, it follows from the differential relationships (173) and (174) that the compatibility condition (163) gives rise to the following important relationship

$$
-\vartheta \varphi=D_{x}^{2} D_{t} \varphi=3 \mu^{2} \eta \varphi,
$$

where the polynomial integro-differential operator

$$
\begin{aligned}
\eta & :=\partial^{-1} \bar{u} \partial^{-3} \bar{u} \partial^{-1}+4 \partial^{-2} \bar{u} \partial^{-1} \bar{u} \partial^{-2} \\
& +2\left(\partial^{-2} \bar{u} \partial^{-2} \bar{u} \partial^{-1}+\partial^{-1} \bar{u} \partial^{-2} \bar{u} \partial^{-2}\right)
\end{aligned}
$$

is skew-symmetric on the functional manifold $M$ and comprises the second compatible Poisson structure for the Ostrovsky-Vakhnenko dynamical system (140).

Now by virtue of the recurrent relationships following from substitution of the asymptotic expansion

$$
\varphi \simeq \sum_{j \in \mathbb{Z}_{+}} \varphi_{j} \xi^{-j}, \xi:=-1 /\left(3 \mu^{2}\right),
$$

into (177), one can determine a new infinite hierarchy of conservations laws for dynamical system (140):

$$
\tilde{\gamma}_{j}:=\int_{0}^{1} \mathrm{~d} s\left(\varphi_{j}[u s], u\right),
$$

for $j \in \mathbb{Z}_{+}$, where

$$
\varphi_{j}=\Lambda^{j} \varphi_{0}, \vartheta \varphi_{0}=0,
$$

and the recursion operator $\Lambda:=\vartheta^{-1} \eta: T^{*}(M) \rightarrow T^{*}(M)$ satisfies the standard Lax representation:

$$
\Lambda_{t}=\left[\Lambda, K^{\prime *}\right] \text {. }
$$

The above results can be formulated as follows.

Proposition 8 The Ostrovsky-Vakhnenko dynamical system (140) allows the standard differential Lax representation (173), (174) and defines on the functional manifold $M$ an integrable bi-Hamiltonian flow with compatible Poisson structures (154) and (178). In particular, this dynamical system possesses an infinite hierarchy of nonlocal conservation laws (180) defined by the gradient elements (181).

Remark 3 It should be noted that the existence of an infinite $\lambda$-powers ordered hierarchy of conservations laws (148) is a typical property $[1,3,10,12]$ of the Lax integrable Hamiltonian systems, which are simultaneously bi-Hamiltonian flows with respect to corresponding compatible Poissonian structures.

Remark 4 It is interesting to observe that our second 
polynomial Poisson structure (178) differs from that obtained recently in [42], which contains rational power factors.

Making use of the differential Expressions (173) and (174), it is easy to construct a slightly different from (175) matrix Lax representation of the Zakharov-Shabat form for the dynamical system (138).

In fact, if one defines the "spectral" parameter $\mu:=1 /(9 \lambda) \in \mathbb{C} \backslash\{0\}$ and new basis elements of the invariant differential ideal (161):

$$
g_{1}:=-3 D_{x} f, g_{2}:=f, g_{3}:=9 \lambda D_{x}^{2} f+u_{x} f,
$$

then relationships (173) and (174) can be rewritten as follows:

$$
D_{t} g=q[u ; \lambda] g, D_{x} g=l[u ; \lambda] g,
$$

where the matrices

$$
\begin{aligned}
& q[u ; \lambda]:=\left(\begin{array}{ccc}
0 & 1 & 0 \\
0 & 0 & 1 \\
\lambda & -u & 0
\end{array}\right), \\
& l[u ; \lambda]:=\left(\begin{array}{ccc}
0 & u_{x} /(3 \lambda) & -1 /(3 \lambda) \\
-1 / 3 & 0 & 0 \\
-u_{x} / 3 & -1 / 3 & 0
\end{array}\right)
\end{aligned}
$$

coincide with those of $[42,43]$ and satisfy the Zakharov-Shabat type compatibility condition:

$$
D_{t} l=[q, l]+D_{x} q-l D_{x} u .
$$

Remark 5 As already mentioned above, the Lax representation (185) of the Ostrovsky-Vakhnenko dynamical system (138) was obtained in [43] by means of a suitable limiting reduction of the Degasperis-Processi equation

$$
u_{t}-u_{x x t}+4 u u_{x}-3 u_{x} u_{x x}-u u_{x x x}=0 .
$$

For convenience, let us rewrite this in the following form:

$$
D_{t} z=-3 z D_{x} u, z=u-D_{x}^{2} u,
$$

where differentiations

$$
D_{t}:=\partial / \partial t+u \partial / \partial x \text { and } D_{x}:=\partial / \partial x
$$

satisfy the Lie-algebraic relationship (77). It is impressive that Equation (187) is itself a special reduction of a new Lax integrable Riemann type hydrodynamic system, proposed and studied (for $s=2$ ) recently in [51]:

$$
D_{t}^{N-1} u=\bar{z}_{x}^{s}, D_{t} \bar{z}=0,
$$

where $s, \quad N \in \mathbb{N}$ are arbitrary natural numbers. Actually, defining $z:=\bar{z}_{x}^{s}$ and $s=3$, from (189) one easily obtains the following dynamical system:

$$
D_{t}^{N-1} u=z, D_{t} z=-3 z D_{x} u
$$

coinciding with the Degasperis-Processi Equation (188) if one makes the identification $\mathrm{z}=u-D_{x}^{2} u$. As a result, we have proved that a function $u \in C^{\infty}\left(\mathbb{R}^{2} ; \mathbb{R}\right)$ that satisfies, for an arbitrary $N \in \mathbb{N}$, the generalized Riemann type hydrodynamical equation

$$
D_{t}^{N-1} u=u-D_{x}^{2} u,
$$

simultaneously solves the Degasperis-Processi equation (187). In particular, for $N=2$, we find that solutions to the Burgers type equation

$$
D_{t} u=u-D_{x}^{2} u
$$

are also solutions to the Degasperis-Processi Equation (187). This means, in particular, that the reduction procedure in [43] can also be applied to the Lax integrable Riemann type hydrodynamic system (189), giving rise to a related Lax representation for the Ostrovsky-Vakhnenko dynamical system (138).

\section{Conclusions}

We have considered the standard canonically symplectic phase space $M:=T^{*}(\tilde{\mathcal{G}})$, generated by the centrally extended basis manifold to be an affine loop Lie algebra $\tilde{\mathcal{G}}$ on the circle $\mathbb{S}^{1}$. Subject to the standard Hamiltonian Lie algebra $\tilde{\mathcal{G}}$-action on $M$, with respect which the symplectic structure on $M$ is invariant, we have constructed the corresponding momentum mapping and carried out the standard Marsden-Weinstein reduction of the manifold $M$ upon the reduced phase space $\bar{M}_{\xi}$ endowed with the reduced Poisson bracket $\{\cdot, \cdot\}_{\xi}$. This allows to construct on the phase space $\bar{M}_{\xi}$ mutually commuting vector fields which are equivalent to nonlinear dynamical systems possessing an infinite hierarchy of commuting conservation laws. Moreover, these commuting vector fields on $\bar{M}_{\xi}$ realize exactly their corresponding Lax representations.

In addition, we have detailed analysis of commutation properties for the related flows on the basis manifold making it possible to define a suitable $D$-structure on the Lie algebra $\tilde{\mathcal{G}}$, intimately related to the corresponding classical $R$-structure on $\tilde{\mathcal{G}}$, generated by the reduced Poisson bracket on the phase space $\bar{M}_{\xi}$. As a by-product of our analysis we proved that these $R$ - and $D$-structures are completely equivalent to a suitably generalized classical Lie-Poisson-Adler-Kostant-SymesKirillov-Berezin structure on the adjoint space $\hat{\mathcal{G}}^{*}$. We also derived the determining Equation for the $D$-structure, classifying the generalized Lax integrable nonlinear dynamical systems on the reduced phase space $\bar{M}_{\xi}$, whose respectively defined $R$-structures are not necessary both antisymmetric and local, as shown in [22,24] by means of another approach. It is also worth mentioning that the reduction scheme devised in this work can be 
applied to the centrally extended algebra of pseudo-differential operators and affine loop algebras on the circle $\mathbb{S}^{1}$.

A new differential-algebraic approach, elaborated in [9] for revisiting the integrability analysis of generalized Riemann type hydrodynamical Equation (73), made it possible to prove the Lax integrability of new nonlinear Hamiltonian dynamical systems representing Riemann type hydrodynamic Equations (80), (87) and (91). In particular, the integrability prerequisites of these dynamical system, such as compatible Poissonian structures, an infinite hierarchy of conservation laws and related Lax representation have been constructed by means of both the symplectic gradient-holonomic approach $[10,12,48]$ and innovative differential-algebraic tools devised recently $[8,9,35]$ for analyzing the integrability of a special infinite hierarchy of Riemann type hydrodynamic systems. It is also quite clear from recent research in this area and our work in this paper that the dynamical system (80) is a Lax integrable bi-Hamiltonian flow for arbitrary integers $N \in \mathbb{N}$. This is perhaps most readily verified by means of the differential-algebraic approach, which was devised and successfully applied here for the cases $N=2$ and 3 .

Making use of the differential-algebraic approach, we have also re-derived the Lax representation for the Ostrovsky-Vakhnenko Equation (138) and constructed the related simple compatible polynomial Poisson structures.

As we have seen in the course of this investigation, perhaps the most important lesson that one can derive from this approach is the following: If an investigation of a given nonlinear Hamiltonian dynamical system via the gradient-holonomic method indicates (but does not necessarily prove) that the system is Lax integrable, then its Lax representation can often be shown to exist and then successfully derived by means of a suitably constructed invariant differential ideal $\mathcal{I}\{u\}$ of the ring $\mathcal{K}\{u\}$ in accordance with the differential-algebraic approach developed here for the integrability analysis of the Riemann hydrodynamical system. Consequently, when it comes applying this lesson to the investigation of other nonlinear dynamical systems, it is natural to start with systems that are known to be Lax integrable and to try to identify and characterize those algebraic structures responsible for the existence of a related finite-dimensional matrix representation for the basic $D_{x}$ - and $D_{t}$-differentiations in a vector space $\mathcal{K}^{p}$ for some finite $p \in \mathbb{Z}_{+}$.

It seems plausible that if one could do this for several classes of Lax integrable dynamical systems, certain patterns in the algebraic structures may be detected that can be used to assemble a more extensive array of symplectic and differential-algebraic tools capable of resolving the question of complete integrability for many other types of nonlinear Hamiltonian dynamical systems. Moreover, if the integrability is established in this manner, the approach should also serve as a means of constructing associated artifacts of the integrability such as Lax representations and hierarchies of mutually commuting invariants. As a particular differential-algebraic problem of interest concerning these matrix representations, one can seek to develop a scheme for the effective construction of functional generators of the corresponding invariant finite-dimensional ideals $\mathcal{I}\{u\} \subset \mathcal{K}\{u\}$ under given differential-algebraic constraints imposed on the $D_{x}$ - and $D_{t}$-differentiations.

We have also demonstrated here that an approach combining the gradient-holonomic method with some recently devised differential-algebraic techniques can be a very effective and efficient way of investigating integrability for a particular class of infinite-dimensional Hamiltonian dynamical systems (generalized Riemann hydrodynamical systems). But a closer look at the specific details of the approach employed here reveals, we believe, that this combination of methods can be adapted to perform effective integrability analysis of a much wider range of dynamical systems.

\section{Acknowledgements}

D.B. acknowledges the National Science Foundation (Grant CMMI-1029809) and A.P. and Y.P. acknowledge the Scientific and Technological Research Council of Turkey (TUBITAK/NASU-111T558 Project) for partial support of their research.

\section{REFERENCES}

[1] L. D. Faddeev and L. A. Takhtadjan, "Hamiltonian Methods in the Theory of Solitons," Springer, Berlin, 2000.

[2] A. G. Reyman and M. A. Semenov-Tyan-Shansky, "Reduction of Hamiltonian Systems, Affine Lie Algebras, and Lax Equations, I, II," Invent. Math, Vol. 54, No. 1, 1979, pp. 81-100 and Vol. 63, No. 3, 1981, pp. 423-432.

[3] M. Blaszak, "Multi-Hamiltonian Theory of Dynamical Systems," Springer, Berlin, 1998.

[4] A. Newell, "Solitons in Mathematics and Physics," SIAM, Philadelphia, 1985. http://dx.doi.org/10.1137/1.9781611970227

[5] S. P. Novikov, "Theory of Solitons," Springer, Berlin, 1984.

[6] A. G. Reyman and M. A. Semenov-Tian-Shansky, "Integrable Systems," The Computer Research Institute Publishing, Moscow-Izhvek, 2003. (in Russian)

[7] A. M. Mikhaylov, A. B. Shabat and R. I. Yamilov, "Extension of the Module of Invertible Transformations. Classification of Integrable Systems," Communications in Mathematical Physics, Vol. 115, No. 1, 1988, pp. 1-19. http://dx.doi.org/10.1007/BF01238850

[8] A. K. Prykarpatsky, O. D. Artemovych, Z. Popowicz and 
M. V. Pavlov, "Differential-Algebraic Integrability Analysis of the Generalized Riemann Type and Korteweg-de Vries Hydrodynamical," Journal of Physics A: Mathematical and Theoretical, Vol. 43, No. 29, 2010, Article ID: 295205.

[9] Y. A. Prykarpatsky, O. D. Artemovych, M. Pavlov and A. K. Prykarpatsky, "The Differential-Algebraic and Bi-Hamiltonian Integrability Analysis of the Riemann Type Hierarchy Revisited," Journal of Mathematical Physics, Vol. 53, 2012, Article ID: 103521.

[10] D. Blackmore, A. K. Prykarpatsky and V. Hr Samoylenko, "Nonlinear Dynamical Systems of Mathematical Physics: Spectral and Differential-Geometrical Integrability Analysis,” World Scientific, New Jersey, 2011.

[11] Y. Mitropolsky, N. Bogolubov Jr., A. Prykarpatsky and V. Samoylenko, "Integrable Dynamical System: Spectral and Differential-Geometric Aspects," Naukova Dunka, Kiev, 1987. (in Russian)

[12] A. Prykarpatsky and I. Mykytyuk, "Algebraic Integrability of Nonlinear Dynamical Systems on Manifolds: Classical and Quantum Aspects," Kluwer Academic Publishers, Dordrecht, The Netherlands, 1998. http://dx.doi.org/10.1007/978-94-011-4994-5

[13] R. Abraham and J. E. Marsden, "Foundations of Mechanics," Benjamin/Cummins Publisher, San Francisco, 1978.

[14] V. I. Arnold, "Mathematical Methods of Classical Mechanics," Springer, Berlin, 1989. http://dx.doi.org/10.1007/978-1-4757-2063-1

[15] M. Adler, "Completely Integrable Systems and Symplectic Action," Journal of Mathematical Physics, Vol. 20, No. 1, 1979, pp. 60-67. http://dx.doi.org/10.1063/1.523963

[16] A. M. Perelomov, "Integrable Systems of Classical Mechanics and Lie Algebras," Nauka Publishing, Moscow, 1990. (in Russian)

[17] N. N. Bogolubov Jr. and Y. A. Prykarpatsky, "The Marsden-Weinstein Reduction Structure of Integrable Dynamical Systems and a Generalized Exactly Solvable Quantum Superradiance Model," International Journal of Modern Physics B, Vol. 28, No. 1, 2012, pp. 237-245.

[18] R. V. Samulyak, "Generalized Dicke Type Dynamical System as the Inverse Nonlinear Schrödinger Equation," Ukrainian Mathematical Journal, Vol. 47, No. 1, 1995, pp. 149-151. http://dx.doi.org/10.1007/BF01058807

[19] M. A. Semenov-Tian-Shansky, "What Is an R-Matrix?" Functional Analysis and Its Applications, Vol. 17, No. 4, 1983, pp. 259-272. http://dx.doi.org/10.1007/BF01076717

[20] Y. A. Prykarpatsky, A. M. Samoilenko and A. K. Prykarpatsky, "The Geometric Properties of Canonically Reduced Symplectic Spaces with Symmetry, Their Relationship with Structures on Associated Principal Fiber Bundles and Some Applications," Opuscula Mathematica, Vol. 25, No. 2, 2005, pp. 287-298.

[21] F. Calogero and A. Degasperis, "Spectral Transform and Solitons," North-Holland, Amsterdam, 1982.

[22] J. Avan, O. Babelon and M. Talon, "Construction of
Classical $R$-Matrices for the Toda and Calogero Models," Algebra and Analysis, Vol. 6, No. 2, 1994, p. 67.

[23] O. Babelon and C.-M. Viallet, "Hamiltonian Structures and Lax Equations," Physics Letter B, Vol. 237, No. 3-4, 1990, pp. 411-416.

[24] G. E. Arutyunov and P. B. Medvedev, "Generating Equation for $r$-Matrices Related to the Dynamical Systems of Calogero Type," Physics Letter A, Vol. 223, No. 1-2, 1996, pp. 66-74.

http://dx.doi.org/10.1016/S0375-9601(96)00719-0

[25] E. K. Sklyanin, "Quantum Variant of the Inverse Scattering Transform Method," Proceedings of LOMI 95, Leningrad, 15-20 January 1980, pp. 55-128. (in Russian)

[26] S. A. Tsyplyaev, "Commutation Relations for Transition Matrix in Classical and Quantum Inverse Scattering Method," Theoretical and Mathematical Physics, Vol. 48, No. 1, 1981, pp. 24-33. (in Russian) http://dx.doi.org/10.1007/BF01037981

[27] T. Crespo and Z. Hajto, "Algebraic Groups and Differential Galois Theory. Graduate Studies in Mathematics Series," American Mathematical Society Publisher, Providence, 2011.

[28] I. Kaplanski, "Introduction to Differential Algebra," Hermann, Paris, 1957.

[29] E. R. Kolchin, "Differential Algebra and Algebraic Groups," Academic Press, New York, 1973.

[30] J. F. Ritt, "Differential Algebra," AMS-Colloqium Publications, New York, 1966.

[31] J.-A. Weil, "Introduction to Differential Algebra and Differential Galois Theory," CIMPA-UNESCO-Vietnam Lectures, Hanoi, 2001.

[32] J. Golenia, M. Pavlov, Z. Popowicz and A. Prykarpatsky, "On a Nonlocal Ostrovsky-Whitham Type Dynamical System, Its Riemann Type Inhomogenious Regularizations and Their Integrability," SIGMA 6, 2010, pp. 1-13.

[33] G. Wilson, "On the Quasi-Hamiltonian Formalism of the KdV Equation,” Physics Letter, Vol. 132, No. 8-9, 1988, pp. 445-450.

[34] L. Brunelli and A. Das, "On an Integrable Hierarchy Derived from the Isentropic Gas Dynamics," Journal of Mathematical Physics, Vol. 45, No. 7, 2004, p. 2633. http://dx.doi.org/10.1063/1.1756699

[35] J. Golenia, N. N. Bogolubov Jr., Z. Popowicz, M. V. Pavlov and A. K. Prykarpatsky, "A New Riemann Type Hydrodynamical Hierarchy and Its Integrability Analysis," 2009. http://publications.ictp.it

[36] M. Pavlov, "The Gurevich-Zybin System," Journal of Physics A: Mathematical and General, Vol. 38, No. 17, 2005, pp. 3823-3840. http://dx.doi.org/10.1088/0305-4470/38/17/008

[37] Z. Popowicz and A. K. Prykarpatsky, "The Non-Polynomial Conservation Laws and Integrability Analysis of Generalized Riemann Type Hydrodynamical Equations," Nonlinearity, Vol. 23, No. 10, 2010, pp. 2517-2537. http://dx.doi.org/10.1088/0951-7715/23/10/010

[38] Y. Prykarpatsky, "Finite Dimensional Local and Nonlocal Reductions of One Type Hydrodynamic Systems," Re- 
ports on Mathematical Physics, Vol. 50, No. 3, 2002, pp. 349-360.

http://dx.doi.org/10.1016/S0034-4877(02)80065-9

[39] A. K. Prykarpatsky and M. M. Prytula, "The GradientHolonomic Integrability Analysis of a Whitham-Type Nonlinear Dynamical Model for a Relaxing Medium with Spatial Memory," Nonlinearity, Vol. 19, No. 9, 2006, pp. 2115-2122. http://dx.doi.org/10.1088/0951-7715/19/9/007

[40] J. P. Wang, "The Hunter-Saxton Equation: Remarkable Structures of Symmetries and Conserved Densities," Nonlinearity, Vol. 23, No. 8, 2010, pp. 2009-2028. http://dx.doi.org/10.1088/0951-7715/23/8/011

[41] Z. Popowicz, "The Matrix Lax Representation of the Generalized Riemann Equations and Its Conservation Laws," Physics Letter A, Vol. 375, No. 37, 2011, pp. 3268-3272.

[42] J. C. Brunelli and S. Sakovich, "Hamiltonian Structures for the Ostrovsky-Vakhnenko Equation," Communications in Nonlinear Science and Numerical Simulation, Vol. 18, No. 1, 2013, pp. 56-62.

[43] A. Degasperis, D. D. Holm and A. N. W Hone, "A New Integrable Equation with Peakon Solutions," Theoretical and Mathematical Physics, Vol. 133, No. 2, 2002, pp. 1463-1474. http://dx.doi.org/10.1023/A:1021186408422

[44] L. A. Ostrovsky, "Nonlinear Internal Waves in a Rotating Ocean," Okeanologia, Vol. 18, No. 2, 1978, pp. 181-191.
[45] A. A. Vakhnenko, "Solitons in a Nonlinear Model Medium," Journal of Physics A, Vol. 25, No. 15, 1992, pp. 4181-4187.

[46] Y. Wang and Y. Chen, "Integrability of the Modified Generalized Vakhnenko Equation," Journal of Mathematical Physics, Vol. 53, No. 12, 2012, Article ID: 123504. http://dx.doi.org/10.1063/1.4764845

[47] G. B. Whitham, "Linear and Nonlinear Waves," WileyInterscience, New York, 1974.

[48] O. Hentosh, M. Prytula and A. Prykarpatsky, "Differential-Geometric and Lie-Algebraic Foundations of Investigating Nonlinear Dynamical Systems on Functional Manifolds," 2nd Edition, Lviv University Publishing, Lviv, 2006. (in Ukrainian)

[49] E. J. Parkes, "The Stability of Solutions of Vakhnenko's Equation," Journal of Physics A, Vol. 26, No. 22, 1993, pp. 6469-6475.

[50] A. G. Reyman and M. A. Semenov-Tian-Shansky, "The Hamiltonian Structure of Kadomtsev-Petviashvili Type Equations," LOMI Proceedings, Leningrad, 12-17 January 1987, pp. 212-227. (in Russian)

[51] D. Blackmore, Y. A. Prykarpatsky, O. D. Artemowych, D. Orest and A. K. Prykarpatsky, "On the Complete Integrability of a One Generalized Riemann Type Hydrodynamic System," arXiv:1204.0251v1. 